\title{
On a Nonlinear Wave Equation of Kirchhoff-Carrier Type: Linear Approximation and Asymptotic Expansion of Solution in a Small Parameter
}

\author{
Nguyen Huu Nhan, ${ }^{1,2}$ Le Thi Phuong Ngoc, ${ }^{3}$ and Nguyen Thanh Long $\mathbb{D i D}^{2}$ \\ ${ }^{1}$ Dong Nai University, 4 Le Quy Don Str., Tan Hiep District, Bien Hoa City, Vietnam \\ ${ }^{2}$ Department of Mathematics and Computer Science, VNUHCM-University of Science, 227 Nguyen Van Cu Str., \\ Dist. 5, Ho Chi Minh City, Vietnam \\ ${ }^{3}$ University of Khanh Hoa, 01 Nguyen Chanh Str., Nha Trang City, Vietnam
}

Correspondence should be addressed to Nguyen Thanh Long; longnt2@gmail.com

Received 25 June 2017; Accepted 10 December 2017; Published 22 January 2018

Academic Editor: Filippo Cacace

Copyright (C) 2018 Nguyen Huu Nhan et al. This is an open access article distributed under the Creative Commons Attribution License, which permits unrestricted use, distribution, and reproduction in any medium, provided the original work is properly cited.

We consider the Robin-Dirichlet problem for a nonlinear wave equation of Kirchhoff-Carrier type. Using the Faedo-Galerkin method and the linearization method for nonlinear terms, the existence and uniqueness of a weak solution are proved. An asymptotic expansion of high order in a small parameter of a weak solution is also discussed.

\section{Introduction}

In this paper, we consider the following Robin-Dirichlet problem for a nonlinear wave equation of Kirchhoff-Carrier type:

$$
\begin{aligned}
& u_{t t}-\frac{\partial}{\partial x}\left[\mu\left(x, t, \int_{0}^{1} g\left(x, y, t, u(y, t), u_{x}(y, t)\right) d y\right)\right. \\
& \left.\cdot u_{x}\right]=f(x, t), \quad 0<x<1,0<t<T, \\
& u_{x}(0, t)-h_{0} u(0, t)=u(1, t)=0, \\
& u(x, 0)=\widetilde{u}_{0}(x), \\
& u_{t}(x, 0)=\widetilde{u}_{1}(x),
\end{aligned}
$$

where $\mu, f, g, \widetilde{u}_{0}, \widetilde{u}_{1}$ are given functions and $h_{0} \geq 0$ is a given constant.

Equation (1) can be considered as a general equation containing relatively some classical equations; for example, when $g\left(x, y, t, u, u_{x}\right)=u_{x}^{2}, f=0$, (1) has a relation to the Kirchhoff wave equation:

$$
\rho h u_{t t}=\left(P_{0}+\frac{E h}{2 L} \int_{0}^{L}\left|\frac{\partial u}{\partial y}(y, t)\right|^{2} d y\right) u_{x x}
$$

(see [1]). This equation is a generalization of the well-known D'Alembert's wave equation for free vibrations of elastic strings. Kirchhoff's model takes into account the changes in length of the string produced by transverse vibrations. The parameters in (4) have the following meanings: $u$ is the lateral deflection, $L$ is the length of the string, $h$ is the area of the cross section, $E$ is the Young modulus of the material, $\rho$ is the mass density, and $P_{0}$ is the initial tension.

In another case, with $g\left(x, y, t, u, u_{x}\right)=u^{2}, f=0$, (1) contains the form of Carrier equation. In [2], Carrier established the equation modeling the vibration of an elastic string when the changes in tension are not small:

$$
\rho h u_{t t}-\left(1+\frac{E A}{L T_{0}} \int_{0}^{L} u^{2} d x\right) u_{x x}=0
$$


where $u(x, t)$ is the $x$-derivative of the deformation, $T_{0}$ is the tension in the rest position, $E$ is the Young modulus, $A$ is the cross section of a string, $L$ is the length of a string, $\rho$ is the density of a material. Therefore, it is clear that (1) considered here contains (4) and (5) as special cases.

Moreover, with various boundary conditions, the particular forms of (1) have been extensively studied by many authors; for example, we refer to [3-15] and the references given therein. In these works, many interesting results about existence, regularity, asymptotic behavior, asymptotic expansion, and decay of solutions were obtained.

Cavalcanti et al., in [4-7], investigated a series of four papers in which the results of existence, global existence, exponential or uniform decay rates, and asymptotic behavior for Kirchhoff-Carrier models are considered.

In [10], the unique existence and asymptotic expansion of solutions of (1) with $\mu=1$ associated with the boundary conditions

$$
u_{x}(0, t)-h_{0} u(0, t)=u_{x}(1, t)+h_{1} u(1, t)=0
$$

and the initial conditions are also studied.

In [15], de Lima Santos studied the asymptotic behavior of solutions of (1) with $f(x, t) \equiv 0, \mu=\mu(t)$, associated with the Dirichlet boundary condition at $x=0$ and a boundary condition of memory type at $x=1$; that is, $u(1, t)+\int_{0}^{t} g(t-$ s) $\mu(s) u_{x}(1, s) d s=0, t>0$.

In [3], Beilin investigated the existence and uniqueness of a generalized solution for the following wave equation with an integral nonlocal condition

$$
\begin{aligned}
& u_{t t}-\Delta u+c(x, t) u=f(x, t), \\
&(x, t) \in \Omega \times(0, T), \\
& \frac{\partial u}{\partial \eta}+\int_{0}^{t} \int_{\Omega} k(x, \xi, \tau) u(\xi, \tau) d \xi d \tau=0, \\
&(x, t) \in \partial \Omega \times[0, T), \\
& u(x, 0)=\widetilde{u}_{0}(x), \\
& u_{t}(x, 0)=\widetilde{u}_{1}(x),
\end{aligned}
$$$$
x \in \Omega,
$$

where $\Omega$ is a bounded domain in $\mathbb{R}^{N}$ with a smooth boundary, $\eta$ is the unit outward normal on $\partial \Omega$, and $f, \widetilde{u}_{0}, \widetilde{u}_{1}$, $k(x, \xi, \tau)$ are given functions. Nonlocal conditions come up when values of the function on the boundary are connected to values inside the domain. There are various types of nonlocal boundary conditions of integral form for hyperbolic, parabolic, or elliptic equations; the ones were introduced in [3].

The well-posedness and optimal decay rate estimates of the energy associated with the Kirchhoff-Carrier problem with memory

$$
\begin{aligned}
& u^{\prime \prime}-M\left(\|\nabla u(t)\|_{2}^{2}\right) \Delta u+\int_{0}^{t} g(t-s) \Delta u(s) d s=0, \\
& \text { in } \Omega \times \mathbb{R}_{+} \text {, } \\
& u=0, \quad \text { on } \Gamma \times \mathbb{R}_{+}, \\
& u(x, 0)=u_{0}(x), \\
& u_{t}(x, 0)=u_{1}(x),
\end{aligned}
$$

in $\Omega$,

where $\Omega$ is a bounded domain in $\mathbb{R}^{N}$, with a smooth boundary $\partial \Omega:=\Gamma$, are proved in [8].

In [11], the following nonlinear wave equation with initial conditions and boundary conditions of two-point type has been investigated:

$$
\begin{aligned}
u_{t t}-\frac{\partial}{\partial x}\left(\mu(x, t) u_{x}\right)+f\left(u, u_{t}\right) & =F(x, t), \\
& 0<x<1,0<t<T .
\end{aligned}
$$

In [12], by combining the linearization method for the nonlinear term, the Faedo-Galerkin method, and the weak compact method, the existence of a unique weak solution of an initial and boundary value problem for nonlinear wave equation $u_{t t}-(\partial / \partial x)\left(\mu\left(x, t, u,\left\|u_{x}\right\|^{2}\right) u_{x}\right)=F\left(x, t, u, u_{x}, u_{t}\right)$ with the nonhomogeneous boundary conditions is proved.

Very recently, in $[13,14]$, with the same method used in [12], the authors proved the results of existence and uniqueness for the wave equations with nonlinear sources containing the nonlocal terms. In [13], the linearization method together with Taylor's expansion is used for both of the source term and the nonlinear integral in it. These techniques have not been used before.

In the same spirit of [10-14], we establish the local existence and uniqueness for prob. (1)-(3) by using the FaedoGalerkin method and the weak compact method. These results are presented in Section 3. In Section 4, the perturbed solution $u_{\varepsilon}(x, t)$ is approximated by the polynomial of $N+1$ degree in a small parameter $\varepsilon$ for the following perturbed equation:

$$
u_{t t}-\frac{\partial}{\partial x}\left(\mu_{\varepsilon}[u](x, t) u_{x}\right)=f(x, t),
$$

$$
0<x<1,0<t<T \text {, }
$$

associated with (2), (3), where

$$
\begin{aligned}
\mu_{\varepsilon}[u](x, t)= & \mu\left(x, t, \int_{0}^{1} g[u](x, y, t) d y\right) \\
& +\varepsilon \mu_{1}\left(x, t, \int_{0}^{1} g_{1}[u](x, y, t) d y\right), \\
g[u](x, y, t)= & g\left(x, y, t, u(y, t), u_{x}(y, t)\right), \\
g_{1}[u](x, y, t)= & g_{1}\left(x, y, t, u(y, t), u_{x}(y, t)\right) .
\end{aligned}
$$




\section{Preliminaries}

Put $\Omega=(0,1)$ and denote the usual function spaces used in this paper by the notations $L^{p}=L^{p}(\Omega), H^{m}=H^{m}(\Omega)$. Let $\langle\cdot, \cdot\rangle$ be either the scalar product in $L^{2}$ or the dual pairing of a continuous linear functional and an element of a function space. The notation $\|\cdot\|$ stands for the norm in $L^{2},\|\cdot\|_{X}$ is the norm in the Banach space $X$, and $X^{\prime}$ is the dual space of $X$.

We denote $L^{p}(0, T ; X), 1 \leq p \leq \infty$ for the Banach space of real functions $u:(0, T) \rightarrow X$ measurable, such that

$$
\begin{aligned}
\|u\|_{L^{p}(0, T ; X)}=\left(\int_{0}^{T}\|u(t)\|_{X}^{p} d t\right)^{1 / p}<\infty, & \\
& \text { for } 1 \leq p<\infty,
\end{aligned}
$$

$\|u\|_{L^{\infty}(0, T ; X)}=\underset{0<t<T}{\operatorname{ess} \sup }\|u(t)\|_{X} \quad$ for $p=\infty$.

Let $u(t), u^{\prime}(t)=u_{t}(t)=\dot{u}(t), u^{\prime \prime}(t)=u_{t t}(t)=\ddot{u}(t)$, $u_{x}(t)=\nabla u(t), u_{x x}(t)=\Delta u(t)$, denote $u(x, t),(\partial u / \partial t)(x, t)$, $\left(\partial^{2} u / \partial t^{2}\right)(x, t),(\partial u / \partial x)(x, t),\left(\partial^{2} u / \partial x^{2}\right)(x, t)$, respectively.

With $\in C^{k}\left([0,1]^{2} \times \mathbb{R}_{+} \times \mathbb{R}^{2}\right), g=g\left(x, y, t, z_{1}, z_{2}\right)$, we put $D_{1} g=\partial g / \partial x, D_{2} g=\partial g / \partial y, D_{3} g=\partial g / \partial t, D_{i+3} g=\partial g / \partial z_{i}$, with $i=1,2$ and $D^{\beta} g=D_{1}^{\beta_{1}} \cdots D_{5}^{\beta_{5}} g ; \beta=\left(\beta_{1}, \ldots, \beta_{5}\right) \epsilon$ $\mathbb{Z}_{+}^{5},|\beta|=\beta_{1}+\cdots+\beta_{5}=k, D^{(0, \ldots, 0)} g=g$.

Similarly, with $\mu=\mu(x, t, z)$, we also put $D_{1} \mu=$ $\partial \mu / \partial x, D_{2} \mu=\partial \mu / \partial t, D_{3} \mu=\partial \mu / \partial z$.

We shall use the following norm on $H^{1}$ :

$$
\|v\|_{H^{1}}=\left(\|v\|^{2}+\left\|v_{x}\right\|^{2}\right)^{1 / 2} .
$$

We put

$$
\begin{aligned}
V & =\left\{v \in H^{1}: v(1)=0\right\}, \\
a(u, v) & =\int_{0}^{1} u_{x}(x) v_{x}(x) d x+h_{0} u(0) v(0),
\end{aligned}
$$

$\forall u, v \in V$.

$V$ is a closed subspace of $H^{1}$ and on $V$ three norms $\|v\|_{H^{1}}$, $\left\|v_{x}\right\|$, and $\|v\|_{a}=\sqrt{a(v, v)}$ are equivalent norms.

We have the following lemmas, the proofs of which are straightforward and hence we omit the details.

Lemma 1. The imbedding $H^{1} \hookrightarrow C^{0}(\bar{\Omega})$ is compact and

$$
\|v\|_{C^{0}(\bar{\Omega})} \leq \sqrt{2}\|v\|_{H^{1}} \quad \forall v \in H^{1},
$$

where $\|v\|_{C^{0}(\bar{\Omega})}=\sup _{x \in[0,1]}|v(x)|($ see $[16])$.

Lemma 2. Let $h_{0} \geq 0$. The imbedding $V \hookrightarrow C^{0}(\bar{\Omega})$ is compact and

$$
\begin{aligned}
\|v\|_{C^{0}(\bar{\Omega})} & \leq\left\|v_{x}\right\| \leq\|v\|_{a}, \\
\frac{1}{\sqrt{2}}\|v\|_{H^{1}} & \leq\left\|v_{x}\right\| \leq\|v\|_{a} \leq \sqrt{1+h_{0}}\|v\|_{H^{1}},
\end{aligned}
$$

for all $v \in V$.
Lemma 3. Let $h_{0} \geq 0$. Then the symmetric bilinear form $a(\cdot, \cdot)$ defined by (15) is continuous on $V \times V$ and coercive on $V$.

Lemma 4. Let $h_{0} \geq 0$. Then there exists the Hilbert orthonormal base $\left\{\widetilde{w}_{j}\right\}$ of $L^{2}$ consisting of the eigenfunctions $\widetilde{w}_{j}$ corresponding to the eigenvalues $\lambda_{j}$ such that

$$
\begin{gathered}
\lim _{j \rightarrow+\infty} \lambda_{j}=+\infty, \quad 0<\lambda_{1} \leq \lambda_{2} \leq \cdots \leq \lambda_{j} \leq \cdots, \\
a\left(\widetilde{w}_{j}, v\right)=\lambda_{j}\left\langle\widetilde{w}_{j}, v\right\rangle \quad \forall v \in V, \quad j=1,2, \ldots
\end{gathered}
$$

Furthermore, the sequence $\left\{\widetilde{w}_{j} / \sqrt{\lambda_{j}}\right\}$ is also a Hilbert orthonormal base of $V$ with respect to the scalar product $a(\cdot, \cdot)$.

On the other hand, we also have $\widetilde{w}_{j}$ satisfying the following boundary value problem:

$$
\begin{aligned}
-\Delta \widetilde{w}_{j} & =\lambda_{j} \widetilde{w}_{j}, \quad \text { in }(0,1), \\
\widetilde{w}_{j x}(0)-h_{0} \widetilde{w}_{j}(0) & =\widetilde{w}_{j}(1)=0, \quad \widetilde{w}_{j} \in V \cap C^{\infty}(\bar{\Omega}) .
\end{aligned}
$$

The proof of Lemma 4 can be found in ([17], p.87, Theorem (7.7)), with $H=L^{2}$ and $V, a(\cdot, \cdot)$ as defined by (14), (15).

Remark 5. The weak formulation of the initial-boundary value problem (1)-(3) can be given in the following manner: Find $u \in \widetilde{W}=\left\{u \in L^{\infty}\left(0, T ; V \cap H^{2}\right): u_{t} \in L^{\infty}(0, T ; V), u_{t t} \in\right.$ $\left.L^{\infty}\left(0, T ; L^{2}\right)\right\}$, such that $u$ satisfies the following variational equation:

$$
\left\langle u_{t t}(t), w\right\rangle+A[u](t ; u(t), w)=\langle f(t), w\rangle,
$$

for all $w \in V$, a.e., $t \in(0, T)$, together with the initial conditions

$$
\begin{aligned}
u(0) & =\widetilde{u}_{0}, \\
u_{t}(0) & =\widetilde{u}_{1},
\end{aligned}
$$

where, for each $w \in \widetilde{W},\{A[w](t ; \cdot, \cdot)\}_{0 \leq t \leq T}$ is the family of symmetric bilinear forms on $V \times V$ defined by

$$
\begin{aligned}
A[w](t ; u, v)= & \left\langle\mu[w](t) u_{x}, v_{x}\right\rangle \\
& +h_{0} \mu[w](0, t) u(0) v(0),
\end{aligned}
$$

for all $u, v \in V, 0 \leq t \leq T$, with $h_{0} \geq 0$ being given constant, and

$$
\begin{aligned}
\mu[w](x, t) & =\mu\left(x, t, \int_{0}^{1} g[w](x, y, t) d y\right), \\
g[w](x, y, t) & =g\left(x, y, t, w(y, t), w_{x}(y, t)\right) .
\end{aligned}
$$

\section{The Existence and Uniqueness}

Let $T^{*}>0$. We make the following assumptions:

$\left(H_{1}\right)\left(\widetilde{u}_{0}, \widetilde{u}_{1}\right) \in\left(V \cap H^{2}\right) \times V$ satisfying the condition $\widetilde{u}_{0 x}(0)-h_{0} \widetilde{u}_{0}(0)=0$. 
$\left(H_{2}\right) g \in C^{2}\left([0,1]^{2} \times\left[0, T^{*}\right] \times \mathbb{R}^{2}\right)$.

$\left(H_{3}\right) \mu \in C^{2}\left([0,1] \times\left[0, T^{*}\right] \times \mathbb{R}\right)$ and there exists a constant $\mu_{0}>0$ such that $\mu(x, t, z) \geq \mu_{0}$, for all $(x, t, z) \epsilon$ $[0,1] \times\left[0, T^{*}\right] \times \mathbb{R}$.

$\left(H_{4}\right) f, f^{\prime} \in L^{2}\left(Q_{T^{*}}\right)$.

For each $M>0$ given, we set the constants $\bar{K}_{M}(g)$, $\bar{K}_{0}(M, g), \widetilde{K}_{M}(\mu), \widetilde{K}_{0}(M, \mu, g)$, as follows:

$$
\begin{aligned}
& \bar{K}_{M}(g)=\sum_{|\alpha| \leq 2} \bar{K}_{0}\left(M, D^{\alpha} g\right), \\
& \widetilde{K}_{M}(\mu)=\sum_{|\beta| \leq 2} \widetilde{K}_{0}\left(M, D^{\beta} \mu, g\right),
\end{aligned}
$$

where

$$
\begin{aligned}
& \bar{K}_{0}(M, g)=\sup _{\left(x, y, t, z_{1}, z_{2}\right) \in A_{1}(M)}\left|g\left(x, y, t, z_{1}, z_{2}\right)\right|, \\
& \widetilde{K}_{0}(M, \mu, g)=\sup _{(x, t, z) \in A_{2}(g, M)}|\mu(x, t, z)|, \\
& A_{1}(M)=\left\{\left(x, t, y, z_{1}, z_{2}\right): 0 \leq x, y \leq 1,0 \leq t\right. \\
& \left.\quad \leq T^{*}, \max _{1 \leq i \leq 2}\left|z_{i}\right| \leq M\right\}, \\
& A_{2}(g, M)=\left\{(x, t, v): 0 \leq x \leq 1,0 \leq t \leq T^{*},|v|\right. \\
& \left.\quad \leq \bar{K}_{0}(M, g)\right\} .
\end{aligned}
$$

For every $T \in\left(0, T^{*}\right]$ and $M>0$, we put

$$
\begin{aligned}
V_{T} & =\left\{v \in L^{\infty}\left(0, T ; V \cap H^{2}\right): v_{t} \in L^{\infty}(0, T ; V), v_{t t}\right. \\
& \left.\in L^{2}\left(Q_{T}\right)\right\},
\end{aligned}
$$

in which $Q_{T}=\Omega \times(0, T)$.

Then $V_{T}$ is a Banach space with respect to the norm

$$
\begin{aligned}
& \|v\|_{V_{T}} \\
& \quad=\max \left\{\|v\|_{L^{\infty}\left(0, T ; V \cap H^{2}\right)},\left\|v_{t}\right\|_{L^{\infty}(0, T ; V)},\left\|v_{t t}\right\|_{L^{2}\left(Q_{T}\right)}\right\} .
\end{aligned}
$$

(See Lions [18]) We also put

$$
\begin{aligned}
W(M, T) & =\left\{v \in V_{T}:\|v\|_{V_{T}} \leq M\right\}, \\
W_{1}(M, T) & =\left\{v \in W(M, T): v_{t t} \in L^{\infty}\left(0, T ; L^{2}\right)\right\} .
\end{aligned}
$$

Now, we establish the recurrent sequence $\left\{u_{m}\right\}$. The first term is chosen as $u_{0} \equiv \widetilde{u}_{0}$, and supposing that

$$
u_{m-1} \in W_{1}(M, T),
$$

we associate problem (1) with the following problem.
Find $u_{m} \in W_{1}(M, T)(m \geq 1)$ satisfying the linear variational problem

$$
\left\langle u_{m}^{\prime \prime}(t), v\right\rangle+A_{m}\left(t ; u_{m}(t), v\right)=\langle f(t), v\rangle,
$$

$\forall v \in V$,

$$
\begin{aligned}
& u_{m}(0)=\widetilde{u}_{0}, \\
& u_{m}^{\prime}(0)=\widetilde{u}_{1},
\end{aligned}
$$

where

$$
\begin{aligned}
& A_{m}(t ; u, v)=A\left[u_{m-1}\right](t ; u, v) \\
& \quad=\left\langle\mu_{m}(t) u_{x}, v_{x}\right\rangle+h_{0} \mu_{m}(0, t) u(0) v(0),
\end{aligned}
$$

$\forall u, v \in V$

$$
\begin{aligned}
& \mu_{m}(x, t)=\mu\left(x, t, \int_{0}^{1} g\left[u_{m-1}\right](x, y, t) d y\right), \\
& g\left[u_{m-1}\right](x, y, t) \\
& \quad=g\left(x, y, t, u_{m-1}(y, t), \nabla u_{m-1}(y, t)\right) .
\end{aligned}
$$

Theorem 6. Suppose that $\left(H_{1}\right)-\left(H_{4}\right)$ hold. Then, there exist positive constants $M, T>0$ such that the recurrent sequence $\left\{u_{m}\right\}$ is defined by (29)-(31).

Proof. The proof consists of several steps.

Step 1 (the Faedo-Galerkin approximation (introduced by Lions [18])). Consider the basis $\left\{w_{j}\right\}$ for $V$ as in Lemma 4 . Approximate solution of (29)-(31) problem which will be found in form

$$
u_{m}^{(k)}(t)=\sum_{j=1}^{k} c_{m j}^{(k)}(t) w_{j}
$$

where the coefficients $c_{m j}^{(k)}(t)$ satisfy the system of linear differential equations

$$
\begin{aligned}
\left\langle\ddot{u}_{m}^{(k)}(t), w_{j}\right\rangle+A_{m}\left(t ; u_{m}^{(k)}(t), w_{j}\right) & =\left\langle f(t), w_{j}\right\rangle, \\
\quad 1 \leq j \leq k, & \\
u_{m}^{(k)}(0) & =\widetilde{u}_{0 k}, \\
\dot{u}_{m}^{(k)}(0) & =\widetilde{u}_{1 k},
\end{aligned}
$$

where

$$
\begin{aligned}
& \widetilde{u}_{0 k}=\sum_{j=1}^{k} \alpha_{j}^{(k)} w_{j} \longrightarrow \widetilde{u}_{0} \quad \text { strongly in } V \cap H^{2}, \\
& \widetilde{u}_{1 k}=\sum_{j=1}^{k} \beta_{j}^{(k)} w_{j} \longrightarrow \widetilde{u}_{1} \quad \text { strongly in } V .
\end{aligned}
$$


The system of (33) can be rewritten in form

$$
\begin{aligned}
& \ddot{c}_{m j}^{(k)}(t)+\sum_{i=1}^{k} A_{i j}^{(m)}(t) c_{m i}^{(k)}(t)=f_{j}(t), \\
& c_{m j}^{(k)}(0)=\alpha_{j}^{(k)}, \\
& \dot{c}_{m j}^{(k)}(0)=\beta_{j}^{(k)}, \\
& \quad 1 \leq j \leq k,
\end{aligned}
$$

where

$$
\begin{aligned}
A_{i j}^{(m)}(t) & =A_{m}\left(t ; w_{i}, w_{j}\right), \\
f_{j}(t) & =\left\langle f(t), w_{j}\right\rangle,
\end{aligned}
$$

$$
1 \leq i, j \leq k .
$$

By (29), it is not difficult to prove that system (35), (36) has a unique solution $c_{m j}^{(k)}(t), 1 \leq j \leq k$ on interval $[0, T]$, so let us omit the details (see [19]).

Step 2 (a priori estimates). First, we need the following lemma.

Lemma 7. Putting $\mu^{*}=\widetilde{K}_{M}(\mu)\left[1+(1+2 M) \bar{K}_{M}(g)\right]$, one has

(i) $\left|A_{m}(t ; u, v)\right| \leq \widetilde{K}(\mu)\|u\|_{a}\|v\|_{a}$

$$
\forall u, v \in V, 0 \leq t \leq T^{*}
$$

(ii) $A_{m}(t ; v, v) \geq \mu_{0}\|v\|_{a}^{2} \quad \forall v \in V, 0 \leq t \leq T^{*}$,

(iii) $\frac{\partial A_{m}}{\partial t}(t ; u, v)$

$$
=\left\langle\mu_{m}^{\prime}(t) u_{x}, v_{x}\right\rangle+h_{0} \mu_{m}^{\prime}(0, t) u(0) v(0),
$$

$\forall u, v \in V$,

(iv) $\left|\frac{\partial A_{m}}{\partial t}(t ; v, v)\right| \leq \mu^{*}\|v\|_{a}^{2} \quad \forall v \in V, 0 \leq t \leq T^{*}$,

(v) $\frac{d}{d t} A_{m}\left(t ; u_{m}^{(k)}(t), u_{m}^{(k)}(t)\right)$

$$
\begin{aligned}
= & 2 A_{m}\left(t ; u_{m}^{(k)}(t), \dot{u}_{m}^{(k)}(t)\right) \\
& +\frac{\partial A_{m}}{\partial t}\left(t ; u_{m}^{(k)}(t), u_{m}^{(k)}(t)\right) .
\end{aligned}
$$

The proof of Lemma 7 is easy; hence we omit the details. Next, we put

$$
S_{m}^{(k)}(t)=X_{m}^{(k)}(t)+Y_{m}^{(k)}(t)+\int_{0}^{t}\left\|\ddot{u}_{m}^{(k)}(s)\right\|^{2} d s,
$$

where

$$
\begin{aligned}
& X_{m}^{(k)}(t)=\left\|\dot{u}_{m}^{(k)}(t)\right\|^{2}+A_{m}\left(t ; u_{m}^{(k)}(t), u_{m}^{(k)}(t)\right), \\
& Y_{m}^{(k)}(t)=\left\|\dot{u}_{m}^{(k)}(t)\right\|_{a}^{2}+\left\|\sqrt{\mu_{m}(t)} \Delta u_{m}^{(k)}(t)\right\|^{2} .
\end{aligned}
$$

Then, it follows from (33), (37) $)_{(\mathrm{iii}),(\mathrm{v})},(38),(39)$ that

$$
\begin{aligned}
S_{m}^{(k)}(t) & \\
= & S_{m}^{(k)}(0)+2\left\langle\mu_{m x}(0) \tilde{u}_{0 k x}, \Delta \tilde{u}_{0 k}\right\rangle \\
& +2\left\langle f(0), \Delta \tilde{u}_{0 k}\right\rangle \\
& +\int_{0}^{t} d s \int_{0}^{1} \mu_{m}^{\prime}(x, s)\left|\Delta u_{m}^{(k)}(x, s)\right|^{2} d x \\
& +\int_{0}^{t} \frac{\partial A_{m}}{\partial t}\left(s ; u_{m}^{(k)}(s), u_{m}^{(k)}(s)\right) d s \\
& +2 \int_{0}^{t}\left\langle\frac{\partial}{\partial s}\left[\mu_{m x}(s) u_{m x}^{(k)}(s)\right], \Delta u_{m}^{(k)}(s)\right\rangle d s \\
& -2\left\langle\mu_{m x}(t) u_{m x}^{(k)}(t), \Delta u_{m}^{(k)}(t)\right\rangle \\
& +2 \int_{0}^{t}\left\langle f(s), \dot{u}_{m}^{(k)}(s)\right\rangle d s \\
& +2 \int_{0}^{t}\left\langle f^{\prime}(s), \Delta u_{m}^{(k)}(s)\right\rangle d s \\
& +2\left\langle f(0), \Delta \tilde{u}_{0 k}\right\rangle+\sum_{j=1}^{8} I_{j} . \\
& -2\left\langle f(t), \Delta u_{m}^{(k)}(t)\right\rangle+\int_{0}^{t}\left\|\ddot{u}_{m}^{(k)}(s)\right\|^{2} d s \\
& +2\left\langle\mu_{m x}(0) \tilde{u}_{0 k x}, \Delta \tilde{u}_{0 k}\right\rangle \\
& \\
& \\
&
\end{aligned}
$$

We shall estimate the terms $I_{j}$ on the right-hand side of (40) as follows.

First Term $I_{1}$. We note that

$$
\begin{aligned}
\mu_{m}^{\prime}(x, t)= & D_{2} \mu\left[u_{m-1}\right] \\
& +D_{3} \mu\left[u_{m-1}\right] \int_{0}^{1} \frac{\partial g\left[u_{m-1}\right]}{\partial t}(x, y, t) d y
\end{aligned}
$$

where we use the notations

$$
\begin{aligned}
& D_{i} \mu\left[u_{m-1}\right]=D_{i} \mu(x, t, \\
&\left.\int_{0}^{1} g\left(x, y, t, u_{m-1}(y, t), \nabla u_{m-1}(y, t)\right) d y\right), \\
& i=1,2,3,
\end{aligned}
$$

$\frac{\partial g\left[u_{m-1}\right]}{\partial t}(x, y, t)=D_{3} g\left[u_{m-1}\right]+D_{4} g\left[u_{m-1}\right]$

$$
\cdot u_{m-1}^{\prime}(y, t)+D_{5} g\left[u_{m-1}\right] \nabla u_{m-1}^{\prime}(y, t),
$$

$D_{i} g\left[u_{m-1}\right](x, y, t)=D_{i} g\left(x, y, t, u_{m-1}(y, t)\right.$,

$\left.\nabla u_{m-1}(y, t)\right), \quad i=1, \ldots, 5$. 
So, by (24), (25), and (41), we obtain

$$
\left|\mu_{m}^{\prime}(x, t)\right| \leq \mu^{*}
$$

Hence,

$$
\begin{aligned}
I_{1} & =\int_{0}^{t} d s \int_{0}^{1} \mu_{m}^{\prime}(x, s)\left|\Delta u_{m}^{(k)}(x, s)\right|^{2} d x \\
& \leq \frac{\mu^{*}}{\mu_{0}} \int_{0}^{t} S_{m}^{(k)}(s) d s .
\end{aligned}
$$

Second Term $I_{2}$. By Lemma 7 (ii) and (iv), we have

$$
\begin{aligned}
\left|I_{2}\right| & =\left|\int_{0}^{t} \frac{\partial A_{m}}{\partial t}\left(s ; u_{m}^{(k)}(s), u_{m}^{(k)}(s)\right) d s\right| \\
& \leq \mu^{*} \int_{0}^{t}\left\|u_{m}^{(k)}(t)\right\|_{a}^{2} d s \leq \frac{\mu^{*}}{\mu_{0}} \int_{0}^{t} S_{m}^{(k)}(s) d s .
\end{aligned}
$$

Third Term $I_{3}$. The Cauchy-Schwartz inequality leads to

$$
\begin{aligned}
\left|I_{3}\right| & =2\left|\int_{0}^{t}\left\langle\frac{\partial}{\partial s}\left[\mu_{m x}(s) u_{m x}^{(k)}(s)\right], \Delta u_{m}^{(k)}(s)\right\rangle d s\right| \\
& \leq \frac{2}{\sqrt{\mu_{0}}} \int_{0}^{t} J_{m}^{(k)}(s) \sqrt{S_{m}^{(k)}(s)} d s
\end{aligned}
$$

where $J_{m}^{(k)}(s)=\left\|(\partial / \partial s)\left[\mu_{m x}(s) u_{m x}^{(k)}(s)\right]\right\|$.

We shall estimate the term $J_{m}^{(k)}(s)$ as follows.

By $S_{m}^{(k)}(t) \geq\left\|\dot{u}_{m x}^{(k)}(t)\right\|^{2}+\left\|u_{m x}^{(k)}(t)\right\|^{2}$, we have

$$
\begin{aligned}
J_{m}^{(k)}(s) & =\left\|\frac{\partial}{\partial s}\left[\mu_{m x}(s) u_{m x}^{(k)}(s)\right]\right\| \\
\leq & \left\|\mu_{m x}(s)\right\|_{C^{0}(\bar{\Omega})}\left\|\dot{u}_{m x}^{(k)}(s)\right\| \\
& +\left\|\mu_{m x}^{\prime}(s)\right\|\left\|u_{m x}^{(k)}(s)\right\|_{C^{0}(\bar{\Omega})} \\
\leq & \left(\left\|\mu_{m x}(s)\right\|_{C^{0}(\bar{\Omega})}+\sqrt{\frac{1}{\mu_{0}}}\left\|\mu_{m x}^{\prime}(s)\right\|\right) \sqrt{S_{m}^{(k)}(s)} .
\end{aligned}
$$

On the other hand, by $\mu_{m x}(x, t)=D_{1} \mu\left[u_{m-1}\right]+$ $D_{3} \mu\left[u_{m-1}\right] \int_{0}^{1} D_{1} g\left[u_{m-1}\right](x, y, t) d y$, it implies that

$$
\left\|\mu_{m x}(t)\right\|_{C^{0}(\bar{\Omega})} \leq \widetilde{K}_{M}(\mu)\left(1+\bar{K}_{M}(g)\right) .
$$

Similarly, from the following equality

$$
\begin{aligned}
& \mu_{m x}^{\prime}(x, t)=\frac{\partial}{\partial t}\left[\frac{\partial \mu_{m}}{\partial x}(x, t)\right]=D_{2} D_{1} \mu\left[u_{m-1}\right] \\
& +D_{3} D_{1} \mu\left[u_{m-1}\right] \int_{0}^{1} \frac{\partial g\left[u_{m-1}\right]}{\partial t}(x, y, t) d y \\
& +\left(D_{2} D_{3} \mu\left[u_{m-1}\right]\right. \\
& \left.+D_{3}^{2} \mu\left[u_{m-1}\right] \int_{0}^{1} \frac{\partial g\left[u_{m-1}\right]}{\partial t}(x, y, t) d y\right) \\
& \cdot \int_{0}^{1} D_{1} g\left[u_{m-1}\right](x, y, t) d y+D_{3} \mu\left[u_{m-1}\right] \\
& \cdot \int_{0}^{1} \frac{\partial D_{1} g\left[u_{m-1}\right]}{\partial t}(x, y, t) d y \\
& \frac{\partial g\left[u_{m-1}\right]}{\partial t}(x, y, t)=D_{3} g\left[u_{m-1}\right](x, y, t) \\
& +D_{4} g\left[u_{m-1}\right](x, y, t) u_{m-1}^{\prime}(y, t)+D_{5} g\left[u_{m-1}\right] \\
& \cdot(x, y, t) \nabla u_{m-1}^{\prime}(y, t) \text {; } \\
& \frac{\partial D_{1} g\left[u_{m-1}\right]}{\partial t}(x, y, t)=D_{3} D_{1} g\left[u_{m-1}\right](x, y, t) \\
& +D_{4} D_{1} g\left[u_{m-1}\right](x, y, t) u_{m-1}^{\prime}(y, t) \\
& +D_{5} D_{1} g\left[u_{m-1}\right](x, y, t) \nabla u_{m-1}^{\prime}(y, t),
\end{aligned}
$$

we obtain that

$$
\begin{aligned}
& \left\|\mu_{m x}^{\prime}(t)\right\| \leq \widetilde{K}_{M}(\mu)[1 \\
& \left.+\bar{K}_{M}(g) \int_{0}^{1}\left(1+\left\|u_{m-1}^{\prime}(t)\right\|+\left\|\nabla u_{m-1}^{\prime}(t)\right\|\right) d y\right] \\
& +\widetilde{K}_{M}(\mu) \bar{K}_{M}(g)[1 \\
& \left.+\bar{K}_{M}(g) \int_{0}^{1}\left(1+\left\|u_{m-1}^{\prime}(t)\right\|+\left\|\nabla u_{m-1}^{\prime}(t)\right\|\right) d y\right] \\
& +\widetilde{K}_{M}(\mu) \bar{K}_{M}(g) \\
& +\int_{0}^{1}\left(1+\left\|u_{m-1}^{\prime}(t)\right\|+\left\|\nabla u_{m-1}^{\prime}(t)\right\|\right) d y \\
& \quad \leq \widetilde{K}_{M}(\mu)\left[1+2(1+M) \bar{K}_{M}(g)\right. \\
& \left.+2(1+2 M) \bar{K}_{M}^{2}(g)\right] .
\end{aligned}
$$

By (48) and (50), it follows from (47) that

$$
J_{m}^{(k)}(s) \leq \zeta_{1}(M) \sqrt{S_{m}^{(k)}(s)},
$$


where

$$
\begin{aligned}
& \zeta_{1}(M)=\widetilde{K}_{M}(\mu)\left(1+\bar{K}_{M}(g)+\sqrt{\frac{1}{\mu_{0}}}[1\right. \\
& \left.\left.+2(1+M) \bar{K}_{M}(g)+2(1+2 M) \bar{K}_{M}^{2}(g)\right]\right) .
\end{aligned}
$$

Therefore, from (46) and (51), we obtain

$$
I_{3} \leq \sqrt{\frac{2}{\mu_{0}}} \zeta_{1}(M) \int_{0}^{t} S_{m}^{(k)}(s) d s .
$$

Fourth Term $I_{4}$. Applying the Cauchy-Schwartz inequality again, we have

$$
\begin{aligned}
\left|I_{4}\right| & =\left|-2\left\langle\mu_{m x}(t) u_{m x}^{(k)}(t), \Delta u_{m}^{(k)}(t)\right\rangle\right| \\
& \leq \frac{1}{\beta}\left\|\mu_{m x}(t) u_{m x}^{(k)}(t)\right\|^{2}+\frac{\beta}{\mu_{0}} S_{m}^{(k)}(t),
\end{aligned}
$$

for all $\beta>0$. On the other hand, it follows from (51) that

$$
\begin{aligned}
& \left\|\mu_{m x}(t) u_{m x}^{(k)}(t)\right\| \\
& \quad=\left\|\mu_{m x}(0) \nabla \widetilde{u}_{0 k}+\int_{0}^{t} \frac{\partial}{\partial s}\left[\mu_{m x}(s) u_{m x}^{(k)}(s)\right] d s\right\| \\
& \leq\left\|\mu_{m x}(0)\right\|_{C^{0}(\bar{\Omega})}\left\|\nabla \widetilde{u}_{0 k}\right\|+\int_{0}^{t} J_{m}^{(k)}(s) d s \\
& \leq\left\|\mu_{m x}(0)\right\|_{C^{0}(\bar{\Omega})}\left\|\nabla \widetilde{u}_{0 k}\right\|+\zeta_{1}(M) \int_{0}^{t} \sqrt{S_{m}^{(k)}(s)} d s .
\end{aligned}
$$

Hence, we obtain from (54) and (55) that

$$
\begin{aligned}
\left|I_{4}\right| \leq & \frac{\beta}{\mu_{0}} S_{m}^{(k)}(t)+\frac{2}{\beta}\left\|\mu_{m x}(0)\right\|_{C^{0}(\bar{\Omega})}^{2}\left\|\nabla \widetilde{u}_{0 k}\right\|^{2} \\
& +\frac{2}{\beta} T \zeta_{1}^{2}(M) \int_{0}^{t} S_{m}^{(k)}(s) d s .
\end{aligned}
$$

$$
\begin{aligned}
\left|I_{5}\right| & =2\left|\int_{0}^{t}\left\langle f(s), \dot{u}_{m}^{(k)}(s)\right\rangle d s\right| \\
& \leq 2 \int_{0}^{t}\|f(s)\|\left\|\dot{u}_{m}^{(k)}(s)\right\| d s \\
& \leq T\|f\|_{L^{\infty}\left(0, T ; L^{2}\right)}^{2}+\int_{0}^{t} S_{m}^{(k)}(s) d s .
\end{aligned}
$$

Sixth Term $I_{6}$. Similarly, we obtain

$$
\begin{aligned}
\left|I_{6}\right| & =\left|2 \int_{0}^{t}\left\langle f^{\prime}(s), \Delta u_{m}^{(k)}(s)\right\rangle d s\right| \\
& \leq\left\|f^{\prime}\right\|_{L^{2}\left(Q_{T^{*}}\right)}^{2}+\frac{1}{\mu_{0}} \int_{0}^{t} S_{m}^{(k)}(s) d s .
\end{aligned}
$$

Seventh Term $I_{7}$. We have

$$
\begin{aligned}
&\left|I_{7}\right|=\left|-2\left\langle f(t), \Delta u_{m}^{(k)}(t)\right\rangle\right| \\
& \leq \frac{1}{\beta}\|f(t)\|^{2}+\beta\left\|\Delta u_{m}^{(k)}(t)\right\|^{2} \\
& \leq \frac{2}{\beta}\left(\|f(0)\|^{2}+T \int_{0}^{t}\left\|f^{\prime}(s)\right\|^{2} d s\right)+\frac{\beta}{\mu_{0}} S_{m}^{(k)}(t) \\
&=\frac{2}{\beta}\left(\|f(0)\|^{2}+T\left\|f^{\prime}\right\|_{L^{2}\left(Q_{T^{*}}\right)}^{2}\right)+\frac{\beta}{\mu_{0}} S_{m}^{(k)}(t), \\
& \quad \forall \beta>0 .
\end{aligned}
$$

Eighth Term $I_{8}$. We note that $(33)_{1}$ can be rewritten as follows:

$$
\begin{aligned}
& \left\langle\ddot{u}_{m}^{(k)}(t), w_{j}\right\rangle-\left\langle\frac{\partial}{\partial x}\left(\mu_{m}(t) u_{m x}^{(k)}(t)\right), w_{j}\right\rangle \\
& =\left\langle f(t), w_{j}\right\rangle, \quad 1 \leq j \leq k .
\end{aligned}
$$

Hence, it follows after replacing $w_{j}$ with $\ddot{u}_{m}^{(k)}(t)$ and integrating that

$$
\begin{aligned}
I_{8}= & \int_{0}^{t}\left\|\ddot{u}_{m}^{(k)}(s)\right\|^{2} d s \\
\leq & 2 \int_{0}^{t}\left\|\frac{\partial}{\partial x}\left(\mu_{m}(s) u_{m x}^{(k)}(s)\right)\right\|^{2} d s+2 \int_{0}^{t}\|f(s)\|^{2} d s \\
\leq & 2 \int_{0}^{t}\left\|\frac{\partial}{\partial x}\left(\mu_{m}(s) u_{m x}^{(k)}(s)\right)\right\|^{2} d s \\
& +2 T\|f\|_{L^{\infty}\left(0, T^{*} ; L^{2}\right)}^{2} .
\end{aligned}
$$

We estimate the term $\left\|(\partial / \partial x)\left(\mu_{m}(s) u_{m x}^{(k)}(s)\right)\right\|^{2}$. By (48), we obtain

$$
\begin{aligned}
& \left\|\frac{\partial}{\partial x}\left(\mu_{m}(s) u_{m x}^{(k)}(s)\right)\right\|^{2} \leq\left(\left\|\mu_{m x}(s) u_{m x}^{(k)}(s)\right\|\right. \\
& \left.+\left\|\mu_{m}(s) \Delta u_{m}^{(k)}(s)\right\|\right)^{2} \leq 2 \widetilde{K}_{M}(\mu) \\
& \cdot\left(\widetilde{K}_{M}(\mu)\left(1+\bar{K}_{M}(g)\right)^{2}\left\|u_{m x}^{(k)}(s)\right\|^{2}\right. \\
& \left.+\left\|\sqrt{\mu_{m}(s)} \Delta u_{m}^{(k)}(s)\right\|^{2}\right) \leq 2 \widetilde{K}_{M}(\mu)(1 \\
& \left.+\widetilde{K}_{M}(\mu)\left(1+\bar{K}_{M}(g)\right)^{2}\right)\left(\left\|u_{m x}^{(k)}(s)\right\|^{2}\right. \\
& \left.+\left\|\sqrt{\mu_{m}(s)} \Delta u_{m}^{(k)}(s)\right\|^{2}\right) \leq 2 \widetilde{K}_{M}(\mu)(1 \\
& \left.+\widetilde{K}_{M}(\mu)\left(1+\bar{K}_{M}(g)\right)^{2}\right)\left(\frac{1+\mu_{0}}{\mu_{0}}\right) S_{m}^{(k)}(s) .
\end{aligned}
$$

Therefore, by Lemma 7 (ii), (61) and (62), we obtain

$$
I_{8} \leq 2 T\|f\|_{L^{\infty}\left(0, T^{*} ; L^{2}\right)}^{2}+\zeta_{2}(M) \int_{0}^{t} S_{m}^{(k)}(s) d s
$$


where

$$
\begin{aligned}
& \zeta_{2}(M) \\
& =4 \widetilde{K}_{M}(\mu)\left(\frac{1+\mu_{0}}{\mu_{0}}\right)\left[1+\widetilde{K}_{M}(\mu)\left(1+\bar{K}_{M}(g)\right)^{2}\right] .
\end{aligned}
$$

Choosing $\beta>0$, with $2 \beta / \mu_{0} \leq 1 / 2$, it follows from (40), (44), (45), (53), (56)-(59), and (63) that

$$
\begin{aligned}
S_{m}^{(k)}(t) \leq & \widetilde{C}_{0}^{(k)}+2 T\left(\frac{2}{\beta}\left\|f^{\prime}\right\|_{L^{2}\left(Q_{T^{*}}\right)}^{2}+3\|f\|_{L^{\infty}\left(0, T^{*} ; L^{2}\right)}^{2}\right) \\
& +\widetilde{C}_{1}(M, T) \int_{0}^{t} S_{m}^{(k)}(s) d s
\end{aligned}
$$

where

$$
\begin{aligned}
& \widetilde{C}_{0}^{(k)}=\widetilde{C}_{0}^{(k)}\left(\mu, f, g, \widetilde{u}_{0 k}, \widetilde{u}_{1 k}\right)=2 S_{m}^{(k)}(0) \\
& +4\left\langle\mu_{m x}(0) \widetilde{u}_{0 k x}, \Delta \widetilde{u}_{0 k}\right\rangle+4\left\langle f(0), \Delta \widetilde{u}_{0 k}\right\rangle \\
& +\frac{4}{\beta}\left\|\mu_{m x}(0)\right\|_{C^{0}(\bar{\Omega})}^{2}\left\|\nabla \widetilde{u}_{0 k}\right\|^{2}+\frac{4}{\beta}\|f(0)\|^{2} \\
& +2\left\|f^{\prime}\right\|_{L^{2}\left(Q_{T^{*}}\right)}^{2} \\
& \widetilde{C}_{1}(M, T)=2\left[1+\frac{1+2 \mu^{*}}{\mu_{0}}+\frac{2}{\beta} T \zeta_{1}^{2}(M)\right. \\
& \left.+\sqrt{\frac{2}{\mu_{0}}} \zeta_{1}(M)+\zeta_{2}(M)\right] .
\end{aligned}
$$

By means of the convergences in (34), we can deduce the existence of a constant $M>0$ independent of $k$ and $m$ such that

$$
\widetilde{C}_{0}^{(k)}\left(\mu, f, g, \widetilde{u}_{0 k}, \widetilde{u}_{1 k}\right) \leq \frac{1}{2} M^{2} .
$$

So, from $(66)_{2}$, we can choose $T \in\left(0, T^{*}\right]$, such that

$$
\begin{aligned}
& {\left[\frac{1}{2} M^{2}+2 T\left(\frac{2}{\beta}\left\|f^{\prime}\right\|_{L^{2}\left(Q_{T^{*}}\right)}^{2}+3\|f\|_{L^{\infty}\left(0, T^{*} ; L^{2}\right)}^{2}\right)\right]} \\
& \cdot \exp \left(T \widetilde{C}_{1}(M, T)\right) \leq M^{2}, \\
& k_{T}=2 \sqrt{T} M \widetilde{K}_{M}(\mu) \bar{K}_{M}(g)\left[1+\sqrt{2}\left(2+\bar{K}_{M}(g)\right)\right] \\
& \cdot\left(1+\frac{1}{\sqrt{\mu_{0}}}\right) \exp \left(\frac{T\left(\mu_{0}+\mu^{*}\right)}{2 \mu_{0}}\right)<1 .
\end{aligned}
$$

Finally, it follows from (65), (67), and (68) that

$$
\begin{aligned}
S_{m}^{(k)}(t) \leq & M^{2} \exp \left(-T \widetilde{C}_{1}(M, T)\right) \\
& +\widetilde{C}_{1}(M, T) \int_{0}^{t} S_{m}^{(k)}(s) d s .
\end{aligned}
$$

By using Gronwall's Lemma, we deduce from (70) that

$$
\begin{aligned}
S_{m}^{(k)}(t) & \leq M^{2} \exp \left(-T \widetilde{C}_{1}(M, T)\right) \exp \left(t \widetilde{C}_{1}(M, T)\right) \\
& \leq M^{2},
\end{aligned}
$$

for all $t \in[0, T]$, for all $m$ and $k$. Therefore, we have

$$
u_{m}^{(k)} \in W(M, T), \quad \forall m, k
$$

Step 3 (limiting process). From (72), we deduce the existence of a subsequence of $\left\{u_{m}^{(k)}\right\}$ still so denoted, such that

$$
\begin{aligned}
& u_{m}^{(k)} \longrightarrow u_{m} \text { in } L^{\infty}\left(0, T ; V \cap H^{2}\right) \text { weak }^{*}, \\
& \dot{u}_{m}^{(k)} \longrightarrow u_{m}^{\prime} \text { in } L^{\infty}(0, T ; V) \text { weak }^{*}, \\
& \ddot{u}_{m}^{(k)} \longrightarrow u_{m}^{\prime \prime} \text { in } L^{2}\left(Q_{T}\right) \text { weak, } \\
& u_{m} \in W(M, T) .
\end{aligned}
$$

Passing to limit in (33), we have $u_{m}$ satisfying (30), (31) in $L^{2}(0, T)$. On the other hand, it follows from $(30)_{1}$ and $(73)_{4}$ that $u_{m}^{\prime \prime}=(\partial / \partial x)\left(\mu_{m}(t) u_{m x}\right)+f \in L^{\infty}\left(0, T ; L^{2}\right)$; hence $u_{m} \in$ $W_{1}(M, T)$ and the proof of Theorem 6 is complete.

We note that $W_{1}(T)=\left\{v \in L^{\infty}(0, T ; V): v^{\prime} \in\right.$ $\left.L^{\infty}\left(0, T ; L^{2}\right)\right\}$ is a Banach space with respect to the norm (see Lions [18]).

$$
\|v\|_{W_{1}(T)}=\|v\|_{L^{\infty}(0, T ; V)}+\left\|v^{\prime}\right\|_{L^{\infty}\left(0, T ; L^{2}\right)} .
$$

We use the result given in Theorem 6 and the compact imbedding theorems to prove the existence and uniqueness of a weak solution of prob. (1)-(3). Hence, we get the main result in this section as follows.

Theorem 8. Let $\left(H_{1}\right)-\left(H_{4}\right)$ hold. Then one has the following.

(i) Prob. (1)-(3) has a unique weak solution $u \in W_{1}(M, T)$, where the constants $M>0$ and $T>0$ are chosen as in Theorem 6.

(ii) Furthermore, the recurrent sequence $\left\{u_{m}\right\}$ defined by (29)-(30) converges to the solution $u$ of prob. (1)-(3) strongly in $W_{1}(T)$.

And one has the estimate

$$
\left\|u_{m}-u\right\|_{W_{1}(T)} \leq C_{T} k_{T}^{m}, \quad \forall m \in \mathbb{N}
$$

where the constant $k_{T} \in[0,1)$ is defined as in (69) and $C_{T}$ is a constant depending only on $T, h_{0}, f, g, \mu, \widetilde{u}_{0}, \widetilde{u}_{1}$, and $k_{T}$.

Proof.

(a) Existence of the Solution. We shall prove that $\left\{u_{m}\right\}$ is a Cauchy sequence in $W_{1}(T)$. Let $w_{m}=u_{m+1}-u_{m}$. Then $w_{m}$ satisfies the variational problem

$$
\begin{aligned}
& \left\langle w_{m}^{\prime \prime}(t), w\right\rangle+A_{m+1}\left(t ; w_{m}(t), w\right) \\
& \quad=-A_{m+1}\left(t ; u_{m}(t), w\right)+A_{m}\left(t ; u_{m}(t), w\right),
\end{aligned}
$$

$\forall w \in V$

$w_{m}(0)=w_{m}^{\prime}(0)=0$. 


$$
\begin{aligned}
& \text { Note that } \\
& \frac{d}{d t} A_{m+1}\left(t ; w_{m}(t), w_{m}(t)\right) \\
& =2 A_{m+1}\left(t ; w_{m}(t), w_{m}^{\prime}(t)\right) \\
& +\frac{\partial A_{m+1}}{\partial t}\left(t ; w_{m}(t), w_{m}(t)\right), \\
& A_{m+1}\left(t ; u_{m}(t), w_{m}^{\prime}(t)\right)-A_{m}\left(t ; u_{m}(t), w_{m}^{\prime}(t)\right) \\
& =-\left\langle\frac{\partial}{\partial x}\left[\left(\mu_{m+1}(t)-\mu_{m}(t)\right) u_{m x}(t)\right], w_{m}^{\prime}(t)\right\rangle .
\end{aligned}
$$

Taking $w=w_{m}^{\prime}(t)$ in $(76)_{1}$, after integrating in $t$, we get

$$
\begin{aligned}
& Z_{m}(t)=\int_{0}^{t} \frac{\partial A_{m+1}}{\partial t}\left(s ; w_{m}(s), w_{m}(s)\right) d s \\
& +2 \int_{0}^{t}\left\langle\frac{\partial}{\partial x}\left[\left(\mu_{m+1}(s)-\mu_{m}(s)\right) u_{m x}(s)\right],\right. \\
& \left.w_{m}^{\prime}(s)\right\rangle d s \equiv J_{1}+J_{2},
\end{aligned}
$$

where

$$
\begin{aligned}
Z_{m}(t) & =\left\|w_{m}^{\prime}(t)\right\|^{2}+A_{m+1}\left(t ; w_{m}(t), w_{m}(t)\right) \\
& \geq\left\|w_{m}^{\prime}(t)\right\|^{2}+\mu_{0}\left\|w_{m}(t)\right\|_{a}^{2},
\end{aligned}
$$

and the integrals on the right-hand side of (78) are estimated as follows.

First Integral $J_{1}$. By $(37)_{(\mathrm{iv})}$ and (79), we have

$$
\begin{aligned}
\left|J_{1}\right| & \leq \int_{0}^{t}\left|\frac{\partial A_{m+1}}{\partial t}\left(s ; w_{m}(s), w_{m}(s)\right)\right| d s \\
& \leq \frac{\mu^{*}}{\mu_{0}} \int_{0}^{t} Z_{m}(s) d s .
\end{aligned}
$$

Second Integral $J_{2}$. By the inequalities

$$
\begin{aligned}
& \left\|\Delta u_{m}(s)\right\|_{H^{2}} \leq\left\|u_{m}(s)\right\|_{H^{2}} \leq M, \\
& \left\|u_{m x}(s)\right\|_{C^{0}(\bar{\Omega})} \leq \sqrt{2}\left\|u_{m x}(s)\right\|_{H^{1}} \leq \sqrt{2}\left\|u_{m}(s)\right\|_{H^{2}} \\
& \quad \leq \sqrt{2} M,
\end{aligned}
$$$$
\left\|D_{i} \mu\left[u_{m}\right](s)\right\|_{C^{0}(\bar{\Omega})} \leq \widetilde{K}_{M}(\mu), \quad i=1,3,
$$

$$
\begin{aligned}
& \left\|D_{1} g\left[u_{m}\right](s)\right\|_{C^{0}(\bar{\Omega})} \leq \bar{K}_{M}(g), \\
& \left\|\mu_{m+1}(s)-\mu_{m}(s)\right\|_{C^{0}(\bar{\Omega})} \\
& \quad \leq 2 \widetilde{K}_{M}(\mu) \bar{K}_{M}(g)\left\|\nabla w_{m-1}(s)\right\| \\
& \quad \leq 2 \widetilde{K}_{M}(\mu) \bar{K}_{M}(g)\left\|w_{m-1}\right\|_{W_{1}(T)}, \\
& \left\|D_{i} \mu\left[u_{m}\right](s)-D_{i} \mu\left[u_{m-1}\right](s)\right\|_{C^{0}(\bar{\Omega})} \\
& \quad \leq 2 \widetilde{K}_{M}(\mu) \bar{K}_{M}(g)\left\|w_{m-1}\right\|_{W_{1}(T)}, \quad i=1,3, \\
& \left\|D_{1} g\left[u_{m}\right](s)-D_{1} g\left[u_{m-1}\right](s)\right\|_{C^{0}(\bar{\Omega})} \\
& \quad \leq 2 \bar{K}_{M}(g)\left\|w_{m-1}\right\|_{W_{1}(T)}
\end{aligned}
$$

and from the equation

$$
\begin{aligned}
& \frac{\partial}{\partial x}\left[\left(\mu_{m+1}(s)-\mu_{m}(s)\right) \nabla u_{m}(s)\right]=\left(\mu_{m+1}(s)\right. \\
& \left.\quad-\mu_{m}(s)\right) \Delta u_{m}(s)+\left(D_{1} \mu\left[u_{m}\right](s)-D_{1} \mu\left[u_{m-1}\right]\right. \\
& \quad \cdot(s)) u_{m x}(s)+\left[\left(D_{3} \mu\left[u_{m}\right](s)-D_{3} \mu\left[u_{m-1}\right](s)\right)\right. \\
& \left.\quad \cdot \int_{0}^{1} D_{1} g\left[u_{m}\right](x, y, s) d y\right] u_{m x}(s)+\left[D_{3} \mu\left[u_{m-1}\right]\right.
\end{aligned}
$$

$$
\left.\cdot(s) \int_{0}^{1}\left(D_{1} g\left[u_{m}\right]-D_{1} g\left[u_{m-1}\right]\right) d y\right] u_{m x}(s)
$$

we obtain that

$$
\begin{gathered}
\left\|\frac{\partial}{\partial x}\left[\left(\mu_{m+1}(s)-\mu_{m}(s)\right) \nabla u_{m}(s)\right]\right\| \leq 2 M \widetilde{K}_{M}(\mu) \\
\cdot \bar{K}_{M}(g)\left[1+\sqrt{2}\left(2+\bar{K}_{M}(g)\right)\right]\left\|w_{m-1}\right\|_{W_{1}(T)} .
\end{gathered}
$$

This implies that

$$
\begin{aligned}
& \left|J_{2}\right| \leq 2 \mid \int_{0}^{t}\left\langle\frac{\partial}{\partial x}\left[\left(\mu_{m+1}(s)-\mu_{m}(s)\right) \nabla u_{m}(s)\right],\right. \\
& \left.w_{m}^{\prime}(s)\right\rangle d s \mid \leq 4 T M^{2} \widetilde{K}_{M}^{2}(\mu) \bar{K}_{M}^{2}(g)[1 \\
& \left.\quad+\sqrt{2}\left(2+\bar{K}_{M}(g)\right)\right]^{2}\left\|w_{m-1}\right\|_{W_{1}(T)}^{2}+\int_{0}^{t} Z_{m}(s) d s .
\end{aligned}
$$

Combining (78), (80), and (84), we obtain

$$
\begin{aligned}
Z_{m}(t) \leq & 4 T M^{2} \widetilde{K}_{M}^{2}(\mu) \bar{K}_{M}^{2}(g) \\
& \cdot\left[1+\sqrt{2}\left(2+\bar{K}_{M}(g)\right)\right]^{2}\left\|w_{m-1}\right\|_{W_{1}(T)}^{2} \\
& +\frac{\mu_{0}+\mu^{*}}{\mu_{0}} \int_{0}^{t} Z_{m}(s) d s .
\end{aligned}
$$

Using Gronwall's Lemma, we deduce from (85) that

$$
\left\|w_{m}\right\|_{W_{1}(T)} \leq k_{T}\left\|w_{m-1}\right\|_{W_{1}(T)}, \quad \forall m \in \mathbb{N},
$$


where $k_{T} \in(0,1)$ is defined as in (69), which implies that

$$
\begin{array}{r}
\left\|u_{m}-u_{m+p}\right\|_{W_{1}(T)} \leq\left\|u_{0}-u_{1}\right\|_{W_{1}(T)}\left(1-k_{T}\right)^{-1} k_{T}^{m}, \\
\forall m, p \in \mathbb{N} .
\end{array}
$$

It follows that $\left\{u_{m}\right\}$ is a Cauchy sequence in $W_{1}(T)$. Then there exists $u \in W_{1}(T)$ such that

$$
u_{m} \longrightarrow u \text { strongly in } W_{1}(T) \text {. }
$$

Note that $u_{m} \in W_{1}(M, T)$; then there exists a subsequence $\left\{u_{m_{j}}\right\}$ of $\left\{u_{m}\right\}$ such that

$$
\begin{aligned}
& u_{m_{j}} \longrightarrow u \quad \text { in } L^{\infty}\left(0, T ; V \cap H^{2}\right) \text { weak }^{*}, \\
& u_{m_{j}}^{\prime} \longrightarrow u^{\prime} \quad \text { in } L^{\infty}(0, T ; V) \text { weak }^{*}, \\
& u_{m_{j}}^{\prime \prime} \longrightarrow u^{\prime \prime} \quad \text { in } L^{2}\left(Q_{T}\right) \text { weak, } \\
& u \in W(M, T) .
\end{aligned}
$$

We also note that

$$
\begin{aligned}
& \left|\mu_{m}(x, t)-\mu[u](x, t)\right| \\
& \quad \leq 2 \widetilde{K}_{M}(\mu) \bar{K}_{M}(g)\left\|u_{m-1}-u\right\|_{W_{1}(T)}, \\
& \text { a.e. }(x, t) \in Q_{T} .
\end{aligned}
$$

Hence, from (88) and (90), we obtain

$$
\mu_{m} \longrightarrow \mu[u] \quad \text { strongly in } L^{\infty}\left(Q_{T}\right) \text {. }
$$

On the other hand, for all $v \in V$, we have

$$
\begin{aligned}
& \left|A_{m}\left(t ; u_{m}, v\right)-A[u](t ; u, v)\right| \leq\left(1+h_{0}\right) \widetilde{K}_{M}(\mu) \\
& \cdot\left[2 \bar{K}_{M}(g) M\left\|u_{m-1}-u\right\|_{W_{1}(T)}+\left\|u_{m}-u\right\|_{W_{1}(T)}\right] \\
& \cdot\left\|v_{x}\right\| .
\end{aligned}
$$

Hence

$$
\int_{0}^{T}\left(A_{m}\left(t ; u_{m}, v\right)-A[u](t ; u, v)\right) \phi(t) d t \longrightarrow 0
$$

$$
\forall v \in V, \forall \phi \in L^{1}(0, T)
$$

Finally, passing to limit in (30)-(31) as $m=m_{j} \rightarrow \infty$, it is implied from (88), (89) $)_{1,3}$, and (93) that there exists $u \in$ $W(M, T)$ satisfying the equation

$$
\left\langle u^{\prime \prime}(t), w\right\rangle+A[u](t ; u(t), w)=\langle f(t), w\rangle
$$

for all $w \in V$ and the initial conditions

$$
\begin{gathered}
u(0)=\tilde{u}_{0}, \\
u^{\prime}(0)=\tilde{u}_{1} .
\end{gathered}
$$

On the other hand, from the assumptions $\left(\mathrm{H}_{2}\right)-\left(\mathrm{H}_{4}\right)$ we obtain from $(89)_{4},(93)$, and (94) that

$$
u^{\prime \prime}=\frac{\partial}{\partial x}\left(\mu[u](t) u_{x}\right)+f \in L^{\infty}\left(0, T ; L^{2}\right),
$$

and thus we have $u \in W_{1}(M, T)$. The existence result follows.

(b) Uniqueness of the Solution. Let $u_{1}, u_{2} \in W_{1}(M, T)$ be two weak solutions of prob. (1)-(3). Then $u=u_{1}-u_{2}$ satisfies the variational problem

$$
\begin{aligned}
& \left\langle u^{\prime \prime}(t), w\right\rangle+A\left[u_{1}\right](t ; u(t), w) \\
& =-A\left[u_{1}\right]\left(t ; u_{2}(t), w\right)+A\left[u_{2}\right]\left(t ; u_{2}(t), w\right), \\
& \forall w \in V, \\
& u(0)=u^{\prime}(0)=0,
\end{aligned}
$$

where

$$
\begin{aligned}
& A\left[u_{i}\right](t ; u, w) \\
& =\left\langle\mu\left[u_{i}\right](t) u_{x}, w_{x}\right\rangle+h_{0} \mu\left[u_{i}\right](0, t) u(0) w(0), \\
& u, w \in V, \\
& \mu\left[u_{i}\right](x, t) \\
& =\mu\left(x, t, \int_{0}^{1} g\left(x, y, t, u_{i}(y, t), \nabla u_{i}(y, t) d y\right)\right), \\
& i=1,2 \text {. }
\end{aligned}
$$

We take $w=u^{\prime}$ in (97) $)_{1}$ and integrate in $t$ to get

$$
\begin{aligned}
& Z(t)=\int_{0}^{t} \frac{\partial A\left[u_{1}\right]}{\partial t}(s ; u(s), u(s)) d s \\
& +2 \int_{0}^{t}\left\langle\frac{\partial}{\partial x}\left[\left(\mu\left[u_{1}\right](s)-\mu\left[u_{2}\right](s)\right) \nabla u_{2}(s)\right]\right. \\
& \left.u^{\prime}(s)\right\rangle d s
\end{aligned}
$$

where $Z(t)=\left\|u^{\prime}(t)\right\|^{2}+A\left[u_{1}\right](t ; u(t), u(t))$.

Put $\widetilde{Z}_{M}=\mu^{*} / \mu_{0}+\left(2 / \sqrt{\mu_{0}}\right) M \widetilde{K}_{M}(\mu) \bar{K}_{M}(g)[1+\sqrt{2}(2+$ $\left.\left.\bar{K}_{M}(g)\right)\right]$, and then it follows from (99) that

$$
Z(t) \leq \widetilde{Z}_{M} \int_{0}^{t} Z(s) d s .
$$

By Gronwall's Lemma, we deduce $Z(t)=0$; that is, $u_{1} \equiv$ $u_{2}$. Theorem 8 is proved completely.

\section{Asymptotic Expansion of the Solution with respect to a Small Parameter}

In this section, let $\left(H_{1}\right)-\left(H_{4}\right)$ hold. We make more the following assumptions:

$$
\left(H_{2}^{\prime}\right) g_{1} \in C^{2}\left([0,1]^{2} \times \mathbb{R}_{+} \times \mathbb{R}^{2}\right)
$$


$\left(H_{3}^{\prime}\right) \mu_{1} \in C^{2}\left([0,1] \times \mathbb{R}_{+} \times \mathbb{R}\right)$ and $\mu_{1}(x, t, z) \geq 0$, for all $(x$, $t, z) \in[0,1] \times \mathbb{R}_{+} \times \mathbb{R}$.

We consider the following perturbed problem, where $\varepsilon$ is a small parameter such that $|\varepsilon| \leq 1$ :

$$
\begin{aligned}
u_{t t}-\frac{\partial}{\partial x}\left[\mu_{\varepsilon}[u](x, t) u_{x}\right] & =f(x, t), \\
0<x<1,0<t<T, & \\
u_{x}(0, t)-h_{0} u(0, t) & =u(1, t)=0, \\
u(x, 0) & =\widetilde{u}_{0}(x), \\
u_{t}(x, 0) & =\widetilde{u}_{1}(x),
\end{aligned}
$$

where

$$
\begin{aligned}
\mu_{\varepsilon}[u](x, t)= & \mu\left(x, t, \int_{0}^{1} g[u](x, y, t) d y\right) \\
& +\varepsilon \mu_{1}\left(x, t, \int_{0}^{1} g_{1}[u](x, y, t) d y\right), \\
g[u](x, y, t)= & g\left(x, y, t, u(y, t), u_{x}(y, t)\right), \\
g_{1}[u](x, y, t)= & g_{1}\left(x, y, t, u(y, t), u_{x}(y, t)\right) .
\end{aligned}
$$

By Theorem 8 , problem $\left(P_{\varepsilon}\right)$ has a unique weak solution $u_{\varepsilon}$ depending on $\varepsilon$, satisfying $u_{\varepsilon} \in W_{1}(M, T)$, in which $M, T$ are independent of $\varepsilon$; these constants are chosen as in (67), (68), and (69), with $\bar{K}_{M}(g)+\bar{K}_{M}\left(g_{1}\right), \widetilde{K}_{M}(\mu)+\widetilde{K}_{M}\left(\mu_{1}\right)$ stand for $\bar{K}_{M}(g), \widetilde{K}_{M}(\mu)$, respectively.

Moreover, we can prove that the limit $u_{0}$ in suitable function spaces of the family $\left\{u_{\varepsilon}\right\}$ as $\varepsilon \rightarrow 0$ is a unique weak solution of the problem $\left(P_{0}\right)$ (corresponding to $\varepsilon=0$ ) also satisfying $u_{0} \in W_{1}(M, T)$.

We shall study the asymptotic expansion of the solution of the problem $\left(P_{\varepsilon}\right)$ with respect to a small parameter $\varepsilon$.

We use the following notations. For a multi-index $\alpha=$ $\left(\alpha_{1}, \ldots, \alpha_{p}\right) \in \mathbb{Z}_{+}^{N}$ and $x=\left(x_{1}, \ldots, x_{N}\right) \in \mathbb{R}^{N}$, we put

$$
\begin{aligned}
|\alpha| & =\alpha_{1}+\cdots+\alpha_{N}, \\
\alpha ! & =\alpha_{1} ! \cdots \alpha_{N} !, \\
\alpha, \beta & \in \mathbb{Z}_{+}^{N}, \\
\alpha & \leq \beta \Longleftrightarrow \alpha_{i} \leq \beta_{i}, \quad \forall i=1, \ldots, N, \\
x^{\alpha} & =x_{1}^{\alpha_{1}} \cdots x_{N}^{\alpha_{N}} .
\end{aligned}
$$

First, we need the following lemma.

Lemma 9. Let $m, N \in \mathbb{N}$ and $x=\left(x_{1}, \ldots, x_{N}\right) \in \mathbb{R}^{N}, \varepsilon \in \mathbb{R}$. Then

$$
\left(\sum_{i=1}^{N} x_{i} \varepsilon^{i}\right)^{m}=\sum_{k=m}^{m N} P_{k}^{(m)}[N, x] \varepsilon^{k}
$$

where the coefficients $P_{k}^{(m)}[N, x], m \leq k \leq m N$, depend on $x=\left(x_{1}, \ldots, x_{N}\right)$ defined by the formulas

$$
\begin{aligned}
& P_{k}^{(m)}[N, x] \\
& \quad= \begin{cases}x_{k}, & 1 \leq k \leq N, m=1, \\
\sum_{\alpha \in A_{k}^{(m)}(N)} \frac{m !}{\alpha !} x^{\alpha}, & m \leq k \leq m N, m \geq 2,\end{cases} \\
& A_{k}^{(m)}(N)=\left\{\alpha \in \mathbb{Z}_{+}^{N}:|\alpha|=m, \sum_{i=1}^{N} i \alpha_{i}=k\right\} .
\end{aligned}
$$

Proof of Lemma 9. The proof of Lemma 9 is easy; hence we omit the details.

Now, we assume that

$$
\left(H_{2}^{N}\right) g \in C^{N+2}\left([0,1]^{2} \times \mathbb{R}_{+} \times \mathbb{R}^{2}\right), g_{1} \in C^{N+1}\left([0,1]^{2} \times \mathbb{R}_{+} \times\right.
$$
$\mathbb{R}^{2}$ );

$\left(H_{3}^{N}\right) \mu \in C^{N+2}\left([0,1] \times \mathbb{R}_{+} \times \mathbb{R}\right), \mu_{1} \in C^{N+1}\left([0,1] \times \mathbb{R}_{+} \times \mathbb{R}\right)$, $\mu \geq \mu_{0}>0$ and $\mu_{1} \geq 0$, for all $(x, t, z) \in[0,1] \times \mathbb{R}_{+} \times$ $\mathbb{R}$.

We use notations $\mu[u](x, t)=\mu\left(x, t, \int_{0}^{1} g[u](x, y, t) d y\right)$, $g[u](x, y, t)=g\left(x, y, t, u(y, t), u_{x}(y, t)\right)$.

Let $u_{0}$ be a unique weak solution of the problem $\left(P_{0}\right)$ corresponding to $\varepsilon=0$; that is,

$$
\begin{aligned}
u_{0}^{\prime \prime}-\frac{\partial}{\partial x}\left(\mu\left[u_{0}\right] u_{0 x}\right) & =f, \quad 0<x<1,0<t<T, \\
u_{0 x}(0, t)-h_{0} u_{0}(0, t) & =u_{0}(1, t)=0, \\
u_{0}(x, 0) & =\widetilde{u}_{0}(x), \\
u_{0}^{\prime}(x, 0) & =\widetilde{u}_{1}(x),
\end{aligned}
$$

$$
u_{0} \in W_{1}(M, T) .
$$

Let us consider the sequence of the weak solutions $u_{k}, 1 \leq k \leq N$, defined by the following problems:

$$
\begin{aligned}
u_{k}^{\prime \prime}-\frac{\partial}{\partial x}\left(\mu\left[u_{0}\right] u_{k x}\right) & =\widetilde{F}_{k}\left[u_{k}\right], \\
0<x<1,0<t<T, & \\
u_{k x}(0, t)-h_{0} u_{k}(0, t)= & u_{k}(1, t)=0, \\
u_{k}(x, 0)= & u_{k}^{\prime}(x, 0)=0, \\
& u_{k} \in W_{1}(M, T),
\end{aligned}
$$

where $\widetilde{F}_{k}\left[u_{k}\right], 1 \leq k \leq N$, are defined by the formulas

$$
\begin{aligned}
\widetilde{F}_{k} & =\widetilde{F}_{k}\left[u_{k}\right] \\
& =\sum_{i=1}^{k} \frac{\partial}{\partial x}\left[\left(\rho_{i}[N, \mu, g]+\rho_{i-1}\left[N-1, \mu_{1}, g_{1}\right]\right)\right. \\
& \left.\nabla u_{k-i}\right], \quad 1 \leq k \leq N,
\end{aligned}
$$


with $\rho_{k}[N, \mu, g]$, which are defined by the formulas

$$
\begin{aligned}
& \rho_{k}[N, \mu, g] \\
& = \begin{cases}\mu\left[u_{0}\right], & k=0, \\
\sum_{j=1}^{k} \frac{1}{j !} D^{j} \mu\left[u_{0}\right] \Phi_{k}\left[j, N, g, u_{0}, \vec{u}\right], & 1 \leq k \leq N,\end{cases}
\end{aligned}
$$

where $\vec{u}=\left(u_{1}, \ldots, u_{N}\right)$ and

$$
\begin{aligned}
& \Phi_{k}\left[j, N, g, u_{0}, \vec{u}\right]=P_{k}^{(j)}\left[N, \vec{\Psi}\left[N, g, u_{0}, \vec{u}, \nabla \vec{u}\right]\right] \\
& = \begin{cases}\bar{\Psi}_{k}\left[N, g, u_{0}, \vec{u}, \nabla \vec{u}\right], & j=1, \\
\sum_{\alpha \in A_{k}^{(j)}(N)} \frac{j !}{\alpha !} \vec{\Psi}^{\alpha}\left[N, g, u_{0}, \vec{u}, \nabla \vec{u}\right], & j \leq k \leq j N, j \geq 2,\end{cases}
\end{aligned}
$$

with $\quad \vec{\Psi}\left[N, g, u_{0}, \vec{u}, \nabla \vec{u}\right]$

$\left(\bar{\Psi}_{1}\left[N, g, u_{0}, \vec{u}, \nabla \vec{u}\right], \ldots, \bar{\Psi}_{N}\left[N, g, u_{0}, \vec{u}, \nabla \vec{u}\right]\right)$, are defined by

$$
\begin{aligned}
& \bar{\Psi}_{k}\left[N, g, u_{0}, \vec{u}, \nabla \vec{u}\right] \\
& =\sum_{1 \leq|\beta| \leq k} \frac{1}{\beta !} \int_{0}^{1} D^{\beta} g\left[u_{0}\right] \Psi_{k}[\beta, N, \vec{u}, \nabla \vec{u}] d y, \\
& \quad 1 \leq k \leq N, \\
& \Psi_{k}[\beta, N, \vec{u}, \nabla \vec{u}] \quad[N, \vec{u}], \\
& =\sum_{(i, j) \in \widetilde{A}(\beta, N), i+j=k} P_{i}^{\left(\beta_{1}\right)}[N, \vec{u}] P_{j}^{\left(\beta_{2}\right)}[N, \vec{A}(\beta, N) \quad \\
& \quad=\left\{(i, j) \in \mathbb{Z}_{+}^{2}: \beta_{1} \leq i \leq N \beta_{1}, \quad \beta_{2} \leq j \leq N \beta_{2}\right\} .
\end{aligned}
$$

Then, we have the following theorem.

Theorem 10. Let $\left(H_{1}\right),\left(H_{4}\right),\left(H_{2}^{N}\right)$, and $\left(H_{3}^{N}\right)$ hold. Then there exist constants $M>0$ and $T>0$ such that, for every $0 \leq \varepsilon \leq 1$, the problem $\left(P_{\varepsilon}\right)$ has a unique weak solution $u_{\varepsilon} \in W_{1}(M, T)$ satisfying the asymptotic estimation up to order $N+1$ as follows:

$$
\left\|u_{\varepsilon}-\sum_{k=0}^{N} u_{k} \varepsilon^{k}\right\|_{W_{1}(T)} \leq C_{T} \varepsilon^{N+1},
$$

where the functions $u_{k}, 0 \leq k \leq N$, are the weak solutions of the problems $\left(P_{0}\right),\left(\widetilde{P}_{k}\right), 1 \leq k \leq N$, respectively, and $C_{T}$ is a constant depending only on $N, T, \mu, \mu_{1}, f, g, g_{1}, u_{k}, 0 \leq k \leq N$. lemmas.

In order to prove Theorem 10, we need the following

Lemma 11. Let $\rho_{k}[N, \mu, g], 1 \leq k \leq N$, be the functions defined by the formulas (106)-(108). Putting $h=\sum_{k=0}^{N} u_{k} \varepsilon^{k}$, then one has

$$
\mu[h]=\sum_{k=0}^{N} \rho_{k}[N, \mu, g] \varepsilon^{k}+\varepsilon^{N+1} \widehat{R}_{N}\left[\mu, g, u_{0}, \vec{u}, \varepsilon\right],
$$

with $\left\|\widehat{R}_{N}\left[\mu, g, u_{0}, \vec{u}, \varepsilon\right]\right\|_{L^{\infty}\left(0, T ; L^{2}\right)} \leq C$, where $C$ is a constant

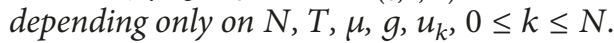

Proof of Lemma 11. In the case of $N=1$, the proof of (110) is easy; hence we omit the details, which we only prove with $N \geq 2$. Putting $h=u_{0}+\sum_{k=1}^{N} u_{k} \varepsilon^{k} \equiv u_{0}+h_{1}$, we rewrite as follows:

$$
\begin{aligned}
\mu[h] & (x, t) \\
& =\mu\left(x, t, \int_{0}^{1} g\left(x, y, t, u_{0}+h_{1}, \nabla u_{0}+\nabla h\right) d y\right) \\
& =\mu\left(x, t, \int_{0}^{1} g\left[u_{0}\right](x, y, t) d y+\xi\right),
\end{aligned}
$$

where $\xi=\int_{0}^{1}\left(g\left[u_{0}+h_{1}\right](x, y, t)-g\left[u_{0}\right](x, y, t)\right) d y$ and $\bar{g}\left[u_{0}\right](x, t)=\int_{0}^{1} g\left(x, y, t, u_{0}(y, t), \nabla u_{0}(y, t)\right) d y$.

By using Taylor's expansion of the function $\mu(x, t$, $\left.\int_{0}^{1} g\left[u_{0}\right](x, y, t) d y+\xi\right)$ around the point $\left[u_{0}\right] \equiv(x, t$, $\left.\int_{0}^{1} g\left[u_{0}\right](x, y, t) d y\right)$ up to order $N+1$, we obtain

$$
\begin{aligned}
\mu[h](x, t)= & \mu\left(x, t, \int_{0}^{1} g\left[u_{0}\right](x, y, t) d y+\xi\right) \\
= & \mu\left[u_{0}\right]+\sum_{j=1}^{N} \frac{1}{j !} D_{3}^{j} \mu\left[u_{0}\right] \xi^{j} \\
& +R_{N}\left[\mu, u_{0}, h_{1}, \xi\right],
\end{aligned}
$$

where

$$
\begin{gathered}
R_{N}\left[\mu, u_{0}, h_{1}, \xi\right]=\frac{1}{N !} \xi^{N+1} \int_{0}^{1}(1-\theta)^{N} D_{3}^{N+1} \mu \\
\cdot\left(x, t, \int_{0}^{1} g\left[u_{0}\right](x, y, t) d y+\theta \xi\right) d \theta \\
=\varepsilon^{N+1} \widehat{R}_{N}^{(1)}\left[\mu, u_{0}, h_{1}, \xi, \varepsilon\right] .
\end{gathered}
$$

Similarly, with $g[h]$, by using Taylor's expansion of the function $g[h](x, y, t)=g\left(x, y, t, u_{0}+h_{1}, \nabla u_{0}+\nabla h_{1}\right)$ around the point $\left[u_{0}\right] \equiv\left(x, y, t, u_{0}, \nabla u_{0}\right)$ up to order $N+1$, we obtain

$$
\begin{aligned}
g[h](x, y, t)= & g\left[u_{0}\right] \\
& +\sum_{1 \leq|\beta| \leq N} \frac{1}{\beta !} D^{\beta} g\left[u_{0}\right] h_{1}^{\beta_{1}}\left(\nabla h_{1}\right)^{\beta_{2}} \\
& +R_{N}\left[g, u_{0}, h_{1}, \varepsilon\right],
\end{aligned}
$$

where

$$
\begin{gathered}
R_{N}\left[g, u_{0}, h_{1}, \varepsilon\right]=\sum_{|\beta|=N+1} \frac{N+1}{\beta !} \int_{0}^{1}(1-\theta)^{N} \\
\cdot D^{\beta} g\left(x, y, t, u_{0}+\theta h_{1}, \nabla u_{0}+\theta \nabla h_{1}\right) \\
\cdot h_{1}^{\beta_{1}}\left(\nabla h_{1}\right)^{\beta_{2}} d \theta=\varepsilon^{N+1} R_{N}^{(1)}\left[\varepsilon, g, u_{0}, h_{1}\right],
\end{gathered}
$$


$\beta=\left(\beta_{1}, \beta_{2}\right) \in \mathbb{Z}_{+}^{2},|\beta|=\beta_{1}+\beta_{2}, \beta !=\beta_{1} ! \beta_{2} !, D^{\beta} g=D_{4}^{\beta_{1}} D_{5}^{\beta_{2}} g$, $D^{\beta} g\left[u_{0}\right]=D^{\beta} g\left(x, y, t, u_{0}, \nabla u_{0}\right)$.

Note, by formula (103), we get

$$
h_{1}^{\beta_{1}}=\left(\sum_{k=1}^{N} u_{k} \varepsilon^{k}\right)^{\beta_{1}}=\sum_{i=\beta_{1}}^{N \beta_{1}} P_{i}^{\left(\beta_{1}\right)}[N, \vec{u}] \varepsilon^{i},
$$

where $\vec{u}=\left(u_{1}, \ldots, u_{N}\right)$

Similarly, with $\left(\nabla h_{1}\right)^{\gamma_{2}}$, we also have

$$
\left(\nabla h_{1}\right)^{\beta_{2}}=\left(\sum_{k=1}^{N} \nabla u_{k} \varepsilon^{k}\right)^{\beta_{2}}=\sum_{j=\beta_{2}}^{N \beta_{2}} P_{j}^{\left(\beta_{2}\right)}[N, \nabla \vec{u}] \varepsilon^{j},
$$

where $\nabla \vec{u}=\left(\nabla u_{1}, \ldots, \nabla u_{N}\right)$.

Hence, we deduce from (116), (117) that

$$
\begin{aligned}
h_{1}^{\beta_{1}}\left(\nabla h_{1}\right)^{\beta_{2}}= & \sum_{k=|\beta|}^{N} \Psi_{k}[\beta, N, \vec{u}, \nabla \vec{u}] \varepsilon^{k} \\
& +\sum_{k=N+1}^{N|\beta|} \Psi_{k}[\beta, N, \vec{u}, \nabla \vec{u}] \varepsilon^{k},
\end{aligned}
$$

where

$$
\begin{aligned}
\Psi_{k} & {[\beta, N, \vec{u}, \nabla \vec{u}] } \\
= & \sum_{(i, j) \in \widetilde{A}(\beta, N), i+j=k} P_{i}^{\left(\beta_{1}\right)}[N, \vec{u}] P_{j}^{\left(\beta_{2}\right)}[N, \nabla \vec{u}], \\
\widetilde{A} & (\beta, N) \\
& =\left\{(i, j) \in \mathbb{Z}_{+}^{2}: \beta_{1} \leq i \leq N \beta_{1}, \beta_{2} \leq j \leq N \beta_{2}\right\} .
\end{aligned}
$$

Hence, it follows from (114), (118) that

$g[h]$

$$
=g\left[u_{0}\right]
$$

$$
+\sum_{1 \leq|\beta| \leq N} \frac{1}{\beta !} D^{\beta} g\left[u_{0}\right] \sum_{k=|\beta|}^{N} \Psi_{k}[\beta, N, \vec{u}, \nabla \vec{u}] \varepsilon^{k}
$$$$
+\sum_{1 \leq|\beta| \leq N} \frac{1}{\beta !} D^{\beta} g\left[u_{0}\right] \sum_{k=N+1}^{N|\beta|} \Psi_{k}[\beta, N, \vec{u}, \nabla \vec{u}] \varepsilon^{k}
$$$$
+\varepsilon^{N+1} R_{N}^{(1)}\left[\varepsilon, g, u_{0}, h_{1}\right]
$$$$
=g\left[u_{0}\right]
$$$$
+\sum_{k=1}^{N} \sum_{1 \leq|\beta| \leq k} \frac{1}{\beta !} D^{\beta} g\left[u_{0}\right] \Psi_{k}[\beta, N, \vec{u}, \nabla \vec{u}] \varepsilon^{k}
$$$$
+\varepsilon^{N+1} R_{N}^{(2)}\left[\varepsilon, g, u_{0}, h_{1}, \vec{u}\right] \text {, }
$$

where

$$
\begin{aligned}
\varepsilon^{N+1} & R_{N}^{(2)}\left[\varepsilon, g, u_{0}, h_{1}, \vec{u}\right] \\
= & \sum_{1 \leq|\beta| \leq N} \frac{1}{\beta !} D^{\beta} g\left[u_{0}\right] \sum_{k=N+1}^{N|\beta|} \Psi_{k}[\beta, N, \vec{u}, \nabla \vec{u}] \varepsilon^{k} \\
& +\varepsilon^{N+1} R_{N}^{(1)}\left[\varepsilon, g, u_{0}, h_{1}\right] .
\end{aligned}
$$

Therefore

$$
\begin{aligned}
\xi & =\int_{0}^{1}\left(g[h](x, y, t)-g\left[u_{0}\right](x, y, t)\right) d y \\
& =\sum_{k=1}^{N}\left(\sum_{1 \leq|\beta| \leq k} \frac{1}{\beta !} \int_{0}^{1} D^{\beta} g\left[u_{0}\right] \Psi_{k}[\beta, N, \vec{u}, \nabla \vec{u}] d y\right) \\
& \cdot \varepsilon^{k}+\varepsilon^{N+1} \int_{0}^{1} R_{N}^{(2)}\left[\varepsilon, g, u_{0}, h_{1}, \vec{u}\right] d y \\
& =\sum_{k=1}^{N} \bar{\Psi}_{k}\left[N, g, u_{0}, \vec{u}, \nabla \vec{u}\right] \varepsilon^{k} \\
& +\varepsilon^{N+1} R_{N}^{(3)}\left[\varepsilon, g, u_{0}, h_{1}, \vec{u}\right],
\end{aligned}
$$

with

$$
\bar{\Psi}_{k}\left[N, g, u_{0}, \vec{u}, \nabla \vec{u}\right]
$$$$
=\sum_{1 \leq|\beta| \leq k} \frac{1}{\beta !} \int_{0}^{1} D^{\beta} g\left[u_{0}\right] \Psi_{k}[\beta, N, \vec{u}, \nabla \vec{u}] d y,
$$

$$
1 \leq k \leq N \text {, }
$$

$R_{N}^{(3)}\left[\varepsilon, g, u_{0}, h_{1}, \vec{u}\right]=\int_{0}^{1} R_{N}^{(2)}\left[\varepsilon, g, u_{0}, h_{1}, \vec{u}\right] d y$.

On the other hand, we also have

$$
\begin{aligned}
\xi^{j} & =\left(\sum_{k=1}^{N} \bar{\Psi}_{k}\left[N, g, u_{0}, \vec{u}, \nabla \vec{u}\right] \varepsilon^{k}\right. \\
& \left.+\varepsilon^{N+1} R_{N}^{(3)}\left[\varepsilon, g, u_{0}, h_{1}, \vec{u}\right]\right)^{j} \\
& =\left(\sum_{k=1}^{N} \bar{\Psi}_{k}\left[N, g, u_{0}, \vec{u}, \nabla \vec{u}\right] \varepsilon^{k}\right)^{j} \\
& +\varepsilon^{N+1} R_{N}^{(4)}\left[\varepsilon, j, g, u_{0}, h_{1}, \vec{u}, \nabla \vec{u}\right] \\
& =\sum_{k=j}^{j N} P_{k}^{(j)}\left[N, \vec{\Psi}\left[N, g, u_{0}, \vec{u}, \nabla \vec{u}\right]\right] \varepsilon^{k} \\
& +\varepsilon^{N+1} R_{N}^{(4)}\left[\varepsilon, j, g, u_{0}, h_{1}, \vec{u}, \nabla \vec{u}\right] \\
& \equiv \sum_{k=j}^{j N} \Phi_{k}\left[j, N, g, u_{0}, \vec{u}\right] \varepsilon^{k} \\
& +\varepsilon^{N+1} R_{N}^{(4)}\left[\varepsilon, j, g, u_{0}, h_{1}, \vec{u}, \nabla \vec{u}\right],
\end{aligned}
$$


where

$$
\begin{aligned}
& \Phi_{k}\left[j, N, g, u_{0}, \vec{u}\right]=P_{k}^{(j)}\left[N, \vec{\Psi}\left[N, g, u_{0}, \vec{u}, \nabla \vec{u}\right]\right] \\
& =\left\{\begin{array}{c}
\bar{\Psi}_{k}\left[N, g, u_{0}, \vec{u}, \nabla \vec{u}\right], \\
\sum_{\alpha \in A_{k}^{(j)}(N)} \frac{j !}{\alpha !} \vec{\Psi}^{\alpha}\left[N, g, u_{0}, \vec{u}, \nabla \vec{u}\right], \quad j \leq k \leq j N, j \geq 2,
\end{array}\right. \\
& A_{k}^{(j)}(N)=\left\{\alpha \in \mathbb{Z}_{+}^{N}:|\alpha|=j, \sum_{i=1}^{N} i \alpha_{i}=k\right\},
\end{aligned}
$$

with $\vec{\Psi}\left[N, g, u_{0}, \vec{u}, \nabla \vec{u}\right]=\left(\bar{\Psi}_{1}\left[N, g, u_{0}, \vec{u}, \nabla \vec{u}\right], \ldots, \bar{\Psi}_{N}[N\right.$, $\left.\left.g, u_{0}, \vec{u}, \nabla \vec{u}\right]\right)$, which are defined by (108).

Decompose the sum $\sum_{k=j}^{j N}$ into the sum of two sums $\sum_{k=j}^{N}$ and $\sum_{k=N+1}^{j N}$; therefore, we deduce from (124) that

$$
\begin{aligned}
\xi^{j}= & \sum_{k=j}^{N} \Phi_{k}\left[j, N, g, u_{0}, \vec{u}\right] \varepsilon^{k} \\
& +\varepsilon^{N+1} R_{N}^{(5)}\left[\varepsilon, j, g, u_{0}, \vec{u}\right],
\end{aligned}
$$

where

$$
\begin{aligned}
\varepsilon^{N+1} & R_{N}^{(5)}\left[\varepsilon, j, g, u_{0}, \vec{u}\right] \\
= & \sum_{k=N+1}^{j N} \Phi_{k}\left[j, N, g, u_{0}, \vec{u}\right] \varepsilon^{k} \\
& +\varepsilon^{N+1} R_{N}^{(4)}\left[\varepsilon, j, g, u_{0}, h_{1}, \vec{u}, \nabla \vec{u}\right]
\end{aligned}
$$

Hence, it follows from (112) and (124) that

$$
\begin{aligned}
& \mu[h]=\mu\left[u_{0}\right] \\
& +\sum_{1 \leq j \leq N} \frac{1}{j !} D^{j} \mu\left[u_{0}\right] \sum_{k=j}^{N} \Phi_{k}\left[j, N, g, u_{0}, \vec{u}\right] \varepsilon^{k} \\
& +\varepsilon^{N+1}\left(\sum_{1 \leq j \leq N} \frac{1}{j !} D^{j} \mu\left[u_{0}\right] R_{N}^{(5)}\left[\varepsilon, j, g, u_{0}, h_{1}, \vec{u}\right]\right. \\
& \left.+\widehat{R}_{N}^{(1)}\left[\mu, u_{0}, h_{1}, \xi, \varepsilon\right]\right)=\mu\left[u_{0}\right] \\
& +\sum_{k=1}^{N}\left(\sum_{1 \leq j \leq k} \frac{1}{j !} D^{j} \mu\left[u_{0}\right] \Phi_{k}\left[j, N, g, u_{0}, \vec{u}\right]\right) \varepsilon^{k} \\
& +\varepsilon^{N+1} \widehat{R}_{N}\left[\mu, g, u_{0}, \vec{u}, \varepsilon\right]=\sum_{k=0}^{N} \rho_{k}[N, \mu, g] \varepsilon^{k} \\
& +\varepsilon^{N+1} \widehat{R}_{N}\left[\mu, g, u_{0}, \vec{u}, \varepsilon\right],
\end{aligned}
$$

where $\rho_{k}[N, \mu, g], 1 \leq k \leq N$, are defined by (106)-(108) and

$$
\begin{aligned}
& \widehat{R}_{N}\left[\mu, g, u_{0}, \vec{u}, \varepsilon\right] \\
& =\sum_{1 \leq j \leq N} \frac{1}{j !} D^{j} \mu\left[u_{0}\right] R_{N}^{(5)}\left[\varepsilon, j, g, u_{0}, h_{1}, \vec{u}\right] \\
& \quad+\widehat{R}_{N}^{(1)}\left[\mu, u_{0}, h_{1}, \xi\right] .
\end{aligned}
$$

By the boundedness of the functions $u_{k}, \nabla u_{k}, 1 \leq k \leq$ $N$, in the function space $L^{\infty}\left(0, T ; L^{2}\right)$, we obtain from (113), (115), (121), (123),$\quad(124)$, and (127) that $\| \widehat{R}_{N}[\mu$, $\left.g, u_{0}, \vec{u}, \varepsilon\right] \|_{L^{\infty}\left(0, T ; L^{2}\right)} \leq C$, where $C$ is a constant depending only on $N, T, \mu, g, u_{k}, 1 \leq k \leq N$. Thus, Lemma 11 is proved.

Remark 12. Lemma 11 is a generalization of the formula contained in ([9], p.262, formula (4.38)) and it is useful to obtain the following Lemma 13. These lemmas are the key to establish the asymptotic expansion of the weak solution $u_{\varepsilon}$ of order $N+1$ in a small parameter $\varepsilon$ as follows.

Let $u=u_{\varepsilon} \in W_{1}(M, T)$ be the unique weak solution of problem $\left(P_{\varepsilon}\right)$. Then $v=u_{\varepsilon}-\sum_{k=0}^{N} u_{k} \varepsilon^{k} \equiv u_{\varepsilon}-h$ satisfies the problem

$$
\begin{aligned}
& v^{\prime \prime}-\frac{\partial}{\partial x}\left(\mu_{\varepsilon}[v+h] v_{x}\right) \\
& =\frac{\partial}{\partial x}\left[\left(\mu_{\varepsilon}[v+h]-\mu_{\varepsilon}[h]\right) h_{x}\right]+E_{\varepsilon}(x, t), \\
& 0<x<1,0<t<T \text {, } \\
& v_{x}(0, t)-h_{0} v(0, t)=v(1, t)=0, \\
& v(x, 0)=v^{\prime}(x, 0)=0,
\end{aligned}
$$

where

$$
\begin{aligned}
E_{\varepsilon}(x, t)= & \frac{\partial}{\partial x}\left[\left(\mu[h]-\mu\left[u_{0}\right]+\varepsilon \mu_{1}[h]\right) h_{x}\right] \\
& -\sum_{k=1}^{N} \widetilde{F}_{k} \varepsilon^{k} .
\end{aligned}
$$

Then, we have the following lemma.

Lemma 13. Let $\left(H_{1}\right),\left(H_{4}\right),\left(H_{2}^{N}\right)$, and $\left(H_{3}^{N}\right)$ hold. Then there exists a constant $C_{*}$ such that

$$
\left\|E_{\varepsilon}\right\|_{L^{\infty}\left(0, T ; L^{2}\right)} \leq C_{*} \varepsilon^{N+1},
$$

where $C_{*}$ is a constant depending only on $N, T, \mu, \mu_{1}, g, g_{1}$, $u_{k}, 1 \leq k \leq N$.

Proof of Lemma 13. In the case of $N=1$, the proof of Lemma 13 is easy; hence we omit the details, which we only prove with $N \geq 2$. 
By using formula (110) for the function $\mu_{1}[h]$ we obtain

$$
\begin{aligned}
\mu_{1}[h]= & \sum_{k=0}^{N-1} \rho_{k}\left[N-1, \mu_{1}, g_{1}\right] \varepsilon^{k} \\
& +\varepsilon^{N} \widehat{R}_{N-1}\left[\mu_{1}, g_{1}, u_{0}, \vec{u}, \varepsilon\right],
\end{aligned}
$$

where $\left\|\widehat{R}_{N-1}\left[\mu_{1}, g_{1}, u_{0}, \vec{u}, \varepsilon\right]\right\|_{L^{\infty}\left(0, T ; L^{2}\right)} \leq C$, with $C$ being a constant depending only on $N, T, \mu_{1}, g_{1}, u_{k}, 0 \leq k \leq N$.

By (133), we rewrite $\varepsilon \mu_{1}[h]$ as follows:

$$
\begin{aligned}
\varepsilon \mu_{1}[h]= & \sum_{k=1}^{N} \rho_{k-1}\left[N-1, \mu_{1}, g_{1}\right] \varepsilon^{k} \\
& +\varepsilon^{N+1} \widehat{R}_{N-1}\left[\mu_{1}, g_{1}, u_{0}, \vec{u}, \varepsilon\right] .
\end{aligned}
$$

Hence, we deduce from (110) and (134) that

$$
\begin{aligned}
& \left(\mu[h]-\mu\left[u_{0}\right]+\varepsilon \mu_{1}[h]\right) h_{x}=\sum_{k=1}^{N} \nabla u_{0}\left(\rho_{k}[N, \mu, g]\right. \\
& \left.+\rho_{k-1}\left[N-1, \mu_{1}, g_{1}\right]\right) \varepsilon^{k} \\
& \quad+\sum_{k=2}^{2 N}\left(\sum_{i, j=1, i+j=k}^{N}\left(\rho_{i}[N, \mu, g]+\rho_{i-1}\left[N-1, \mu_{1}, g_{1}\right]\right)\right. \\
& \left.\quad \nabla u_{j}\right) \varepsilon^{k}+\varepsilon^{N+1} \widetilde{R}_{N}^{(1)}\left[\mu, g, \mu_{1}, g_{1}, u_{0}, \vec{u}, \varepsilon\right]
\end{aligned}
$$

where

$$
\begin{aligned}
& \widetilde{R}_{N}^{(1)}\left[\mu, g, \mu_{1}, g_{1}, u_{0}, \vec{u}, \varepsilon\right] \\
& \quad=\left(\widehat{R}_{N}\left[\mu, g, u_{0}, \vec{u}, \varepsilon\right]+\widehat{R}_{N-1}\left[\mu_{1}, g_{1}, u_{0}, \vec{u}, \varepsilon\right]\right) h_{x} .
\end{aligned}
$$

We decompose the sum $\sum_{i=2}^{2 N}$ into the sum of two sums $\sum_{i=2}^{N}$ and $\sum_{i=N+1}^{2 N}$; therefore, we deduce from (135) that

$$
\begin{aligned}
& \left(\mu[h]-\mu\left[u_{0}\right]+\varepsilon \mu_{1}[h]\right) h_{x}=\sum_{k=1}^{N} \nabla u_{0}\left(\rho_{k}[N, \mu, g]\right. \\
& \left.+\rho_{k-1}\left[N-1, \mu_{1}, g_{1}\right]\right) \varepsilon^{k} \\
& +\sum_{k=2}^{N}\left(\sum_{i, j=1, i+j=k}^{N}\left(\rho_{i}[N, \mu, g]+\rho_{i-1}\left[N-1, \mu_{1}, g_{1}\right]\right)\right. \\
& \left.\nabla u_{j}\right) \varepsilon^{k} \\
& +\sum_{k=N+1}^{2 N}\left(\sum_{i, j=1, i+j=k}^{N}\left(\rho_{i}[N, \mu, g]+\rho_{i-1}\left[N-1, \mu_{1}, g_{1}\right]\right)\right. \\
& \left.\nabla u_{j}\right) \varepsilon^{k}+\varepsilon^{N+1} \widetilde{R}_{N}^{(1)}\left[\mu, g, \mu_{1}, g_{1}, u_{0}, \vec{u}, \varepsilon\right] \\
& \quad=\sum_{k=1}^{N}\left[\sum_{i=1}^{k}\left(\rho_{i}[N, \mu, g]+\rho_{i-1}\left[N-1, \mu_{1}, g_{1}\right]\right) \nabla u_{k-i}\right] \varepsilon^{k} \\
& +\varepsilon^{N+1} \widetilde{R}_{N}^{(2)}\left[\mu, g, \mu_{1}, g_{1}, u_{0}, \vec{u}, \varepsilon\right],
\end{aligned}
$$

where

$$
\begin{aligned}
& \varepsilon^{N+1} \widetilde{R}_{N}^{(2)}\left[\mu, g, \mu_{1}, g_{1}, u_{0}, \vec{u}, \varepsilon\right] \\
& =\sum_{k=N+1}^{2 N}\left(\sum_{i, j=1, i+j=k}^{N}\left(\rho_{i}[N, \mu, g]+\rho_{i-1}\left[N-1, \mu_{1}, g_{1}\right]\right)\right. \\
& \left.\nabla u_{j}\right) \varepsilon^{k}+\varepsilon^{N+1} \widetilde{R}_{N}^{(1)}\left[\mu, g, \mu_{1}, g_{1}, u_{0}, \vec{u}, \varepsilon\right] .
\end{aligned}
$$

Combining (105), (131), and (137) leads to

$$
E_{\varepsilon}(x, t)=\varepsilon^{N+1} \frac{\partial}{\partial x} \widetilde{R}_{N}^{(2)}\left[\mu, g, \mu_{1}, g_{1}, u_{0}, \vec{u}, \varepsilon\right]
$$

By the boundedness of the functions $u_{k}, \nabla u_{k}, 1 \leq k \leq$ $N$, in the function space $L^{\infty}\left(0, T ; H^{1}\right)$, we obtain from (110), (136), (138), and (139) that

$$
\left\|E_{\varepsilon}\right\|_{L^{\infty}\left(0, T ; L^{2}\right)} \leq C_{*} \varepsilon^{N+1},
$$

where $C_{*}$ is a constant depending only on $N, T, \mu, \mu_{1}, g, g_{1}$, $u_{k}, 1 \leq k \leq N$.

Lemma 13 is proved.

Proof of Theorem 10. Consider the sequence $\left\{v_{m}\right\}$ defined by

$$
\begin{aligned}
& v_{0} \equiv 0 \\
& \begin{aligned}
v_{m}^{\prime \prime} & -\frac{\partial}{\partial x}\left(\mu_{\varepsilon}\left[v_{m-1}+h\right] v_{m x}\right) \\
& =\frac{\partial}{\partial x}\left[\left(\mu_{\varepsilon}\left[v_{m-1}+h\right]-\mu_{\varepsilon}[h]\right) h_{x}\right]+E_{\varepsilon}(x, t), \\
& 0<x<1,0<t<T,
\end{aligned} \\
& v_{m x}(0, t)-h_{0} v_{m}(0, t)=v_{m}(1, t)=0, \\
& v_{m}(x, 0)=v_{m}^{\prime}(x, 0)=0, \quad m \geq 1 .
\end{aligned}
$$

By multiplying two sides of (141) with $v_{m}^{\prime}$ and after integration in $t$, we have

$$
\begin{aligned}
& Z_{m}(t)=2 \int_{0}^{t}\left\langle E_{\varepsilon}(s), v_{m}^{\prime}(s)\right\rangle d s+\int_{0}^{t} \frac{\partial A_{m, \varepsilon}}{\partial t}(s \\
& \left.v_{m}(s), v_{m}(s)\right) d s \\
& +2 \int_{0}^{t}\left\langle\frac{\partial}{\partial x}\left[\left(\mu_{\varepsilon}\left[v_{m-1}+h\right]-\mu_{\varepsilon}[h]\right) h_{x}\right],\right. \\
& \left.v_{m}^{\prime}(s)\right\rangle d s
\end{aligned}
$$


where

$$
\begin{aligned}
& Z_{m}(t)=\left\|v_{m}^{\prime}(t)\right\|^{2}+A_{m, \varepsilon}\left(t ; v_{m}(t), v_{m}(t)\right), \\
& A_{m, \varepsilon}(t ; u, v) \\
& =\left\langle\mu_{m, \varepsilon}(t) u_{x}, v_{x}\right\rangle+h_{0} \mu_{m, \varepsilon}(0, t) u(0) v(0), \\
& \mu_{m, \varepsilon}(x, t) \\
& =\mu\left(x, t, \int_{0}^{1} g\left[v_{m-1}+h\right](x, y, t) d y\right) \\
& +\varepsilon \mu_{1}\left(x, t, \int_{0}^{1} g_{1}\left[v_{m-1}+h\right](x, y, t) d y\right), \\
& g\left[v_{m-1}+h\right](x, y, t) \\
& =g\left(x, y, t, v_{m-1}+h, \nabla v_{m-1}+\nabla h\right), \\
& g_{1}\left[v_{m-1}+h\right](x, y, t) \\
& =g_{1}\left(x, y, t, v_{m-1}+h, \nabla v_{m-1}+\nabla h\right) \text {. }
\end{aligned}
$$$$
u, v \in V,
$$

By using Lemma 13, we deduce from (142) that

$$
\begin{aligned}
& \left\|v_{m}^{\prime}(t)\right\|^{2}+\mu_{0}\left\|v_{m}(t)\right\|_{a}^{2} \leq T C_{*}^{2} \varepsilon^{2 N+2} \\
& +\int_{0}^{t}\left\|v_{m}^{\prime}(s)\right\|^{2} d s \\
& +\int_{0}^{t} \frac{\partial A_{m, \varepsilon}}{\partial t}\left(s ; v_{m}(s), v_{m}(s)\right) d s \\
& +2 \int_{0}^{t}\left\|\frac{\partial}{\partial x}\left[\left(\mu_{\varepsilon}\left[v_{m-1}+h\right]-\mu_{\varepsilon}[h]\right) h_{x}\right]\right\| \\
& \cdot\left\|v_{m}^{\prime}(s)\right\| d s=T C_{*}^{2} \varepsilon^{2 N+2}+\int_{0}^{t}\left\|v_{m}^{\prime}(s)\right\|^{2} d s \\
& +\widehat{J}_{1}+\widehat{J}_{2} .
\end{aligned}
$$

We estimate the integrals on the right-hand side of (144) as follows.

Estimating $\widehat{J}_{1}$. We note that

$$
\begin{gathered}
\mu_{m, \varepsilon}^{\prime}(x, t)=D_{2} \mu\left[v_{m-1}+h\right](x, t)+D_{3} \mu\left[v_{m-1}+h\right] \\
\cdot(x, t) \int_{0}^{1}\left[D_{3} g\left[v_{m-1}+h\right]+D_{4} g\left[v_{m-1}+h\right]\right. \\
\cdot\left(v_{m-1}^{\prime}+h^{\prime}\right)(y, t)+D_{5} g\left[v_{m-1}+h\right] \\
\left.\cdot\left(\nabla v_{m-1}^{\prime}+\nabla h^{\prime}\right)(y, t)\right] d y+\varepsilon D_{2} \mu_{1}\left[v_{m-1}+h\right]
\end{gathered}
$$

$$
\cdot(x, t)+\varepsilon D_{3} \mu_{1}\left[v_{m-1}+h\right](x, t)
$$$$
\cdot \int_{0}^{1}\left[D_{3} g_{1}\left[v_{m-1}+h\right]+D_{4} g_{1}\left[v_{m-1}+h\right]\right.
$$$$
\cdot\left(v_{m-1}^{\prime}+h^{\prime}\right)(y, t)+D_{5} g_{1}\left[v_{m-1}+h\right]
$$$$
\left.\cdot\left(\nabla v_{m-1}^{\prime}+\nabla h^{\prime}\right)(y, t)\right] d y \text {, }
$$

and we have

$$
\left|\mu_{m, \varepsilon}^{\prime}(x, t)\right| \leq \bar{\zeta}_{1},
$$

with $\bar{\zeta}_{1}=\widetilde{K}_{M_{*}}(\mu)\left[1+\bar{K}_{M_{*}}(g)\left(1+2 M_{*}\right)\right]+\widetilde{K}_{M_{*}}\left(\mu_{1}\right)[1+$ $\left.\bar{K}_{M_{*}}\left(g_{1}\right)\left(1+2 M_{*}\right)\right], M_{*}=(N+2) M$.

It follows from (146) that

$$
\begin{aligned}
\left|\widehat{J}_{1}\right| & \leq \int_{0}^{t}\left|\frac{\partial A_{m, \varepsilon}}{\partial t}\left(s ; v_{m}(s), v_{m}(s)\right)\right| d s \\
& \leq \bar{\zeta}_{1} \int_{0}^{t}\left\|v_{m}(s)\right\|_{a}^{2} d s .
\end{aligned}
$$

Estimating $\widehat{J}_{2}$. First, we need an estimation $\|(\partial / \partial x)\left[\left(\mu\left[v_{m-1}+\right.\right.\right.$ $\left.h]-\mu[h]) h_{x}\right] \|$.

$$
\text { We also note that }
$$

$$
\begin{aligned}
& \left\|\mu\left[v_{m-1}+h\right]-\mu[h]\right\|_{C^{0}(\bar{\Omega})} \\
& \quad \leq 2 \widetilde{K}_{M_{*}}(\mu) \bar{K}_{M_{*}}(g)\left\|v_{m-1}\right\|_{W_{1}(T)}, \\
& \left\|D_{i} \mu\left[v_{m-1}+h\right]-D_{i} \mu[h]\right\| \\
& \quad \leq 2 \widetilde{K}_{M_{*}}(\mu) \bar{K}_{M_{*}}(g)\left\|v_{m-1}\right\|_{W_{1}(T)}, \quad i=1,3, \\
& \left\|D_{1} g\left[v_{m-1}+h\right]-D_{1} g[h]\right\| \\
& \quad \leq 2 \bar{K}_{M_{*}}(g)\left\|v_{m-1}\right\|_{W_{1}(T)} .
\end{aligned}
$$

From the equation

$$
\begin{aligned}
& \frac{\partial}{\partial x}\left[\left(\mu\left[v_{m-1}+h\right]-\mu[h]\right) h_{x}\right] \\
& \quad=\left(\mu\left[v_{m-1}+h\right]-\mu[h]\right) h_{x x} \\
& \quad+\left(D_{1} \mu\left[v_{m-1}+h\right]-D_{1} \mu[h]\right) h_{x} \\
& \quad+D_{3} \mu\left[v_{m-1}+h\right] \\
& \quad \cdot\left(\int_{0}^{1}\left(D_{1} g\left[v_{m-1}+h\right]-D_{1} g[h]\right) d y\right) h_{x} \\
& \quad+\left(D_{3} \mu\left[v_{m-1}+h\right]-D_{3} \mu[h]\right)\left(\int_{0}^{1} D_{1} g[h] d y\right) \\
& \quad \cdot h_{x},
\end{aligned}
$$

it follows that

$$
\begin{gathered}
\left\|\frac{\partial}{\partial x}\left[\left(\mu\left[v_{m-1}+h\right]-\mu[h]\right) h_{x}\right]\right\| \\
\leq d\left(\mu, g, M_{*}\right)\left\|v_{m-1}\right\|_{W_{1}(T)},
\end{gathered}
$$


where $d\left(\mu, g, M_{*}\right)=2 M_{*} \widetilde{K}_{M_{*}}(\mu) \bar{K}_{M_{*}}(g)[1+\sqrt{2}(2+$ $\left.\left.\bar{K}_{M_{*}}(g)\right)\right]$.

Next, by $\mu_{\varepsilon}=\mu+\varepsilon \mu_{1}$, it follows that

$$
\left\|\frac{\partial}{\partial x}\left[\left(\mu_{\varepsilon}\left[v_{m-1}+h\right]-\mu_{\varepsilon}[h]\right) h_{x}\right]\right\| \leq \bar{\zeta}_{2}\left\|v_{m-1}\right\|_{W_{1}(T)},
$$

where $\bar{\zeta}_{2}=d\left(\mu, g, M_{*}\right)+d\left(\mu_{1}, g_{1}, M_{*}\right)$.

By (151), we obtain

$\widehat{J_{2}}$

$$
\begin{aligned}
& =2 \int_{0}^{t}\left\|\frac{\partial}{\partial x}\left[\left(\mu_{\varepsilon}\left[v_{m-1}+h\right]-\mu_{\varepsilon}[h]\right) h_{x}\right]\right\|\left\|v_{m}^{\prime}(s)\right\| d s \\
& \leq T \bar{\zeta}_{2}^{2}\left\|v_{m-1}\right\|_{W_{1}(T)}^{2}+\int_{0}^{t}\left\|v_{m}^{\prime}(s)\right\|^{2} d s .
\end{aligned}
$$

Combining (144), (147), and (152), this leads to

$$
\begin{aligned}
Y_{m}(t) \leq & T C_{*}^{2} \varepsilon^{2 N+2}+T \bar{\zeta}_{2}^{2}\left\|v_{m-1}\right\|_{W_{1}(T)}^{2} \\
& +\left(2+\frac{\bar{\zeta}_{1}}{\mu_{0}}\right) \int_{0}^{t} Y_{m}(s) d s
\end{aligned}
$$

where $Y_{m}(t)=\left\|v_{m}^{\prime}(t)\right\|^{2}+\mu_{0}\left\|v_{m}(t)\right\|_{a}^{2}$.

By using Gronwall's Lemma, we deduce from (153) that

$$
\left\|v_{m}\right\|_{W_{1}(T)} \leq \sigma_{T}\left\|v_{m-1}\right\|_{W_{1}(T)}+\delta_{T}(\varepsilon), \quad \forall m \geq 1,
$$

where $\sigma_{T}=\eta_{T} \bar{\zeta}_{2}, \delta_{T}(\varepsilon)=C_{*} \eta_{T} \varepsilon^{N+1}, \eta_{T}=(1+1 /$ $\left.\sqrt{\mu_{0}}\right) \sqrt{T \exp \left[\left(1+\bar{\zeta}_{1} / 2 \mu_{0}\right) T\right]}$.

We can assume that

$$
\sigma_{T}<1, \quad \text { with the suitable constant } T>0 .
$$

We require the following lemma whose proof is immediate.

Lemma 14. Let the sequence $\left\{\gamma_{m}\right\}$ satisfy

$$
\gamma_{m} \leq \sigma \gamma_{m-1}+\delta \quad \forall m \geq 1, \gamma_{0}=0,
$$

where $0 \leq \sigma<1, \delta \geq 0$ are the given constants. Then

$$
\gamma_{m} \leq \frac{\delta}{(1-\sigma)} \quad \forall m \geq 1 .
$$

Applying Lemma 14 with $\gamma_{m}=\left\|v_{m}\right\|_{W_{1}(T)}, \sigma=\sigma_{T}<1$, $\delta=\delta_{T}(\varepsilon)$, it follows from (154) that

$$
\left\|v_{m}\right\|_{W_{1}(T)} \leq \frac{\delta_{T}(\varepsilon)}{1-\sigma_{T}}=C_{T} \varepsilon^{N+1},
$$

where $C_{T}$ is a constant depending only on $T$.

On the other hand, the linear recurrent sequence $\left\{v_{m}\right\}$ defined by (141) converges strongly in the space $W_{1}(T)$ to the solution $v$ of problem (130). Hence, letting $m \rightarrow+\infty$ in (158), we get

$$
\|v\|_{W_{1}(T)} \leq C_{T} \varepsilon^{N+1}
$$

This implies (109).

The proof of Theorem 10 is complete.

\section{Conflicts of Interest}

The authors declare that they have no conflicts of interest.

\section{Authors' Contributions}

All authors contributed equally to this article. They read and approved the final manuscript.

\section{References}

[1] G. R. Kirchhoff, Vorlesungen über Mathematische Physik, Mechanik, Section 29.7, Teuber, Leipzig, Germany, 1876.

[2] G. F. Carrier, "On the non-linear vibration problem of the elastic string," Quarterly of Applied Mathematics, vol. 3, pp. 157-165, 1945.

[3] S. A. Beilin, "On a mixed nonlocal problem for a wave equation," Electronic Journal of Differential Equations, vol. 103, pp. 1-10, 2006.

[4] M. M. Cavalcanti, V. N. Domingos Cavalcanti, J. S. Prates Filho, and J. A. Soriano, "Existence and exponential decay for a Kirchhoff-Carrier model with viscosity," Journal of Mathematical Analysis and Applications, vol. 226, no. 1, pp. 40-60, 1998.

[5] M. M. Cavalcanti, V. N. Domingos Cavalcanti, J. A. Soriano, and J. S. Prates Filho, "Existence and asymptotic behaviour for a degenerate Kirchhoff-Carrier model with viscosity and nonlinear boundary conditions," Revista Matemática Complutense, vol. 14, no. 1, pp. 177-203, 2001.

[6] M. M. Cavalcanti, V. N. Domingos Cavalcanti, and J. A. Soriano, "Global existence and uniform decay rates for the KirchhoffCarrier equation with nonlinear dissipation," Advances in Differential Equations, vol. 6, no. 6, pp. 701-730, 2001.

[7] M. M. Cavalcanti, V. N. Domingos Cavalcanti, J. Ferreira, and R. Benabidallah, "On global solvability and asymptotic behaviour of a mixed problem for a nonlinear degenerate Kirchhoff model in moving domains," Bulletin of the Belgian Mathematical Society-Simon Stevin, vol. 10, no. 2, pp. 179-196, 2003.

[8] A. Guesmia, S. A. Messaoudi, and C. M. Webler, "Wellposedness and optimal decay rates for the viscoelastic Kirchhoff equation," Boletim da Sociedade Paranaense de Matemática, vol. 35, no. 3, pp. 203-224, 2017.

[9] N. T. Long, "On the nonlinear wave equation $u t t-B(t, u 2, u \times 2)$ $u x x=f(x, t, u, u x, u t, u 2, u x 2)$ associated with the mixed homogeneous conditions," Journal of Mathematical Analysis and Applications, vol. 306, no. 1, pp. 243-268, 2005.

[10] N. T. Long and T. N. Diem, "On the nonlinear wave equation $u t t-u x x=f(x, t, u, u x, u t)$ associated with the mixed homogeneous conditions," Nonlinear Analysis: Theory, Methods \& Applications, vol. 29, no. 11, pp. 1217-1230, 1997.

[11] N. T. Long and L. T. Ngoc, "On a nonlinear wave equation with boundary conditions of two-point type," Journal of Mathematical Analysis and Applications, vol. 385, no. 2, pp. 1070-1093, 2012.

[12] L. T. P. Ngoc, N. A. Triet, and N. T. Long, "On a nonlinear wave equation involving the term - $\partial \partial x \mu(x, t, u, u x 2) u x$ : linear approximation and asymptotic expansion of solution in many small parameters," Nonlinear Analysis: Real World Applications, vol. 11, no. 4, pp. 2479-2501, 2010.

[13] N. H. Nhan, L. T. Ngoc, T. M. Thuyet, and N. T. Long, "A RobinDirichlet problem for a nonlinear wave equation with the source term containing a nonlinear integral," Lithuanian Mathematical Journal, vol. 57, no. 1, pp. 80-108, 2017. 
[14] N. H. Nhan, L. T. Ngoc, and N. Long, "Existence and asymptotic expansion of the weak solution for a wave equation with nonlinear source containing nonlocal term," Boundary Value Problems, vol. 1, article 87, 2017.

[15] M. de Lima Santos, "Asymptotic behavior of solutions to wave equations with a memory condition at the boundary," Electronic Journal of Differential Equations, vol. 73, pp. 1-11, 2001.

[16] H. Brezis, Functional Analysis, Sobolev Spaces and Partial Differential Equations, Springer, New York, NY, USA, 2010.

[17] R. E. Showalter, "Hilbert space methods for partial differential equations," Electronic Journal of Differential Equations, Monograph, vol. 1, 1994.

[18] J. L. Lions, Quelques Méthodes de Résolution des Problèmes aux Limites Non Linéaires, Dunod; Gauthier-Villars, Paris, Paris, Farnce, 1969.

[19] E. A. Coddington and N. Levinson, Theory of Ordinary Differential Equations, McGraw-Hill, 1955. 


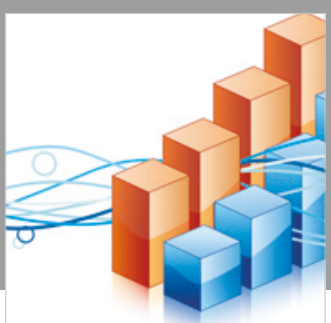

Advances in

Operations Research

\section{-n-m}
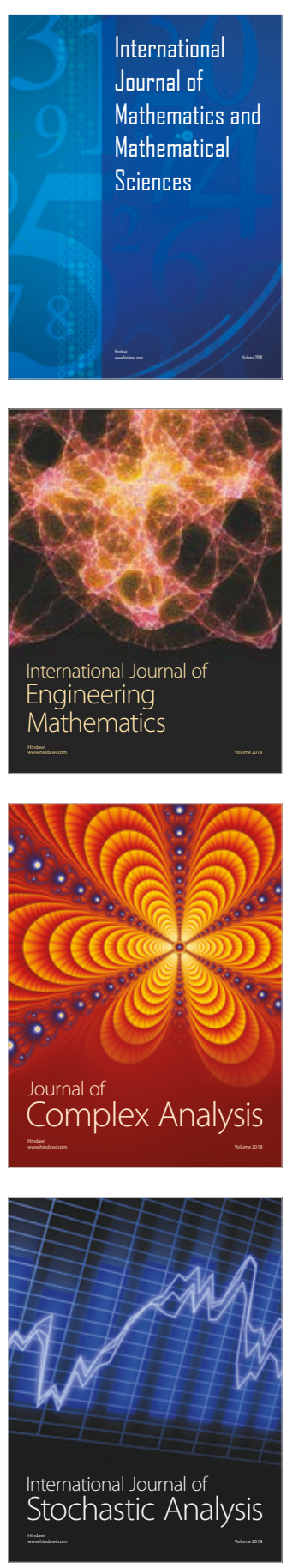
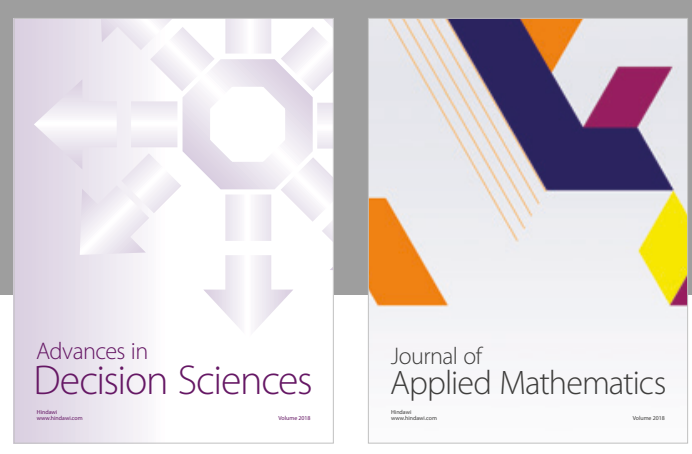

Journal of

Applied Mathematics
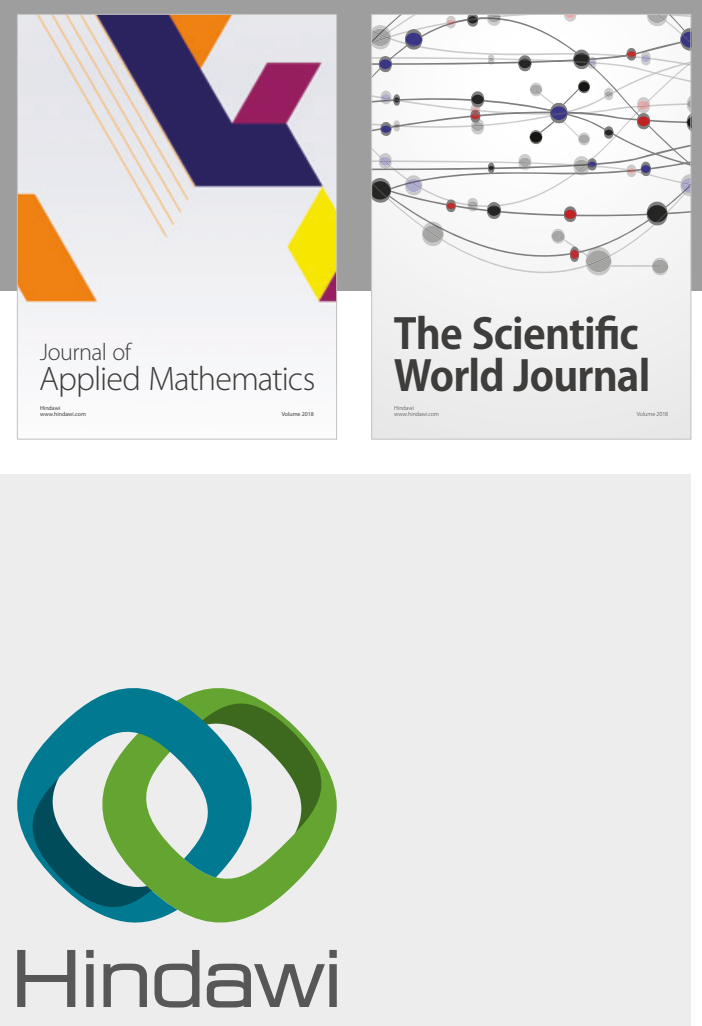

Submit your manuscripts at

www.hindawi.com

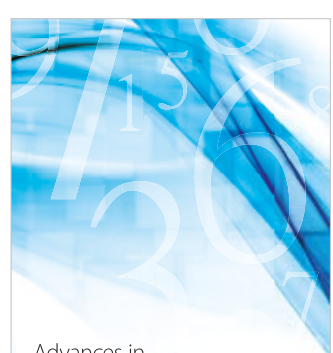

Advances in
Numerical Analysis
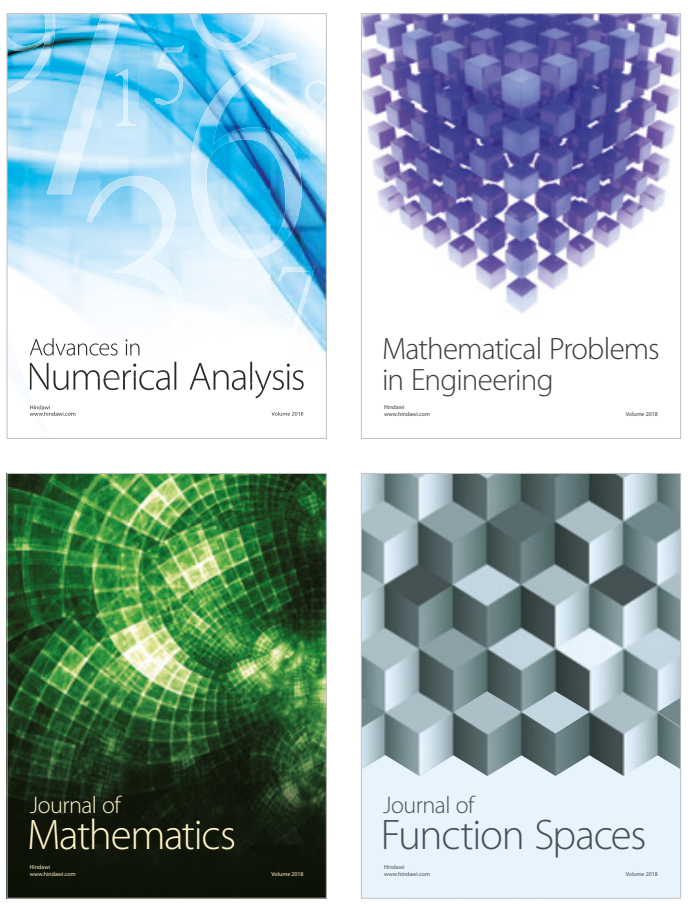

Mathematical Problems in Engineering

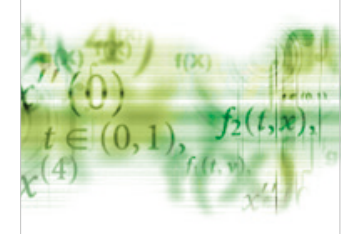

International Journal of

Differential Equations

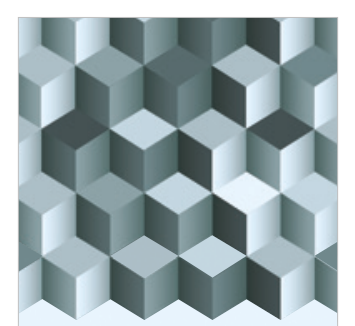

Journal of

Function Spaces

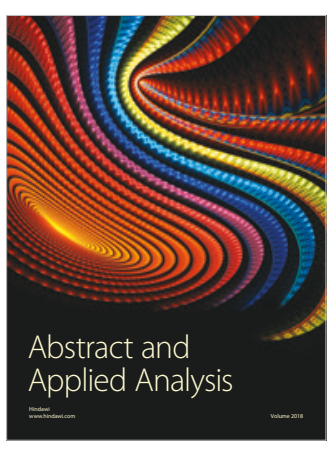

The Scientific

World Journal

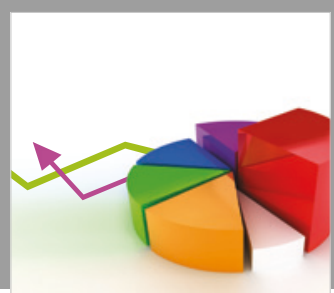

Journal of

Probability and Statistics
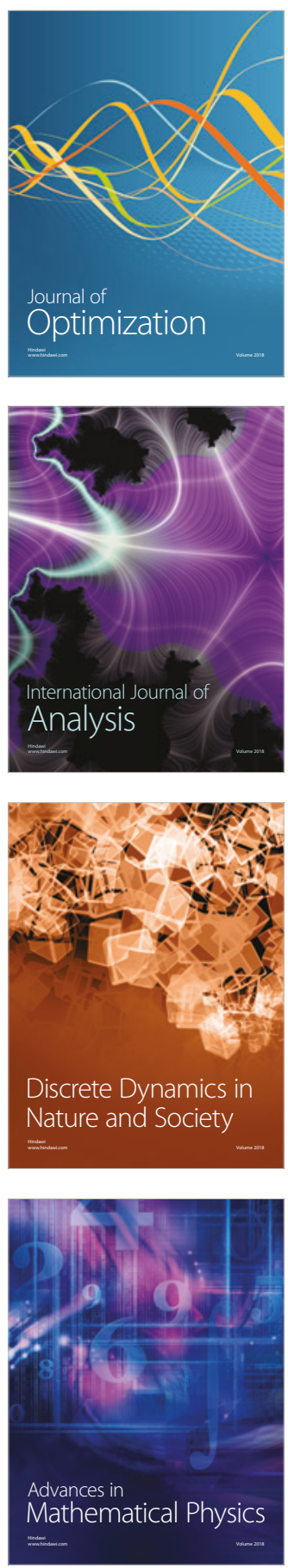